\title{
DYNAMICAL SYSTEMS AND CATEGORIES
}

\author{
G. DIMITROV, F. HAIDEN, L. KATZARKOV, M. KONTSEVICH
}

\begin{abstract}
We study questions motivated by results in the classical theory of dynamical systems in the context of triangulated and $A_{\infty}$-categories. First, entropy is defined for exact endofunctors and computed in a variety of examples. In particular, the classical entropy of a pseudo-Anosov map is recovered from the induced functor on the Fukaya category. Second, the density of the set of phases of a Bridgeland stability condition is studied and a complete answer is given in the case of bounded derived categories of quivers. Certain exceptional pairs in triangulated categories, which we call Kronecker pairs, are used to construct stability conditions with density of phases. Some open questions and further directions are outlined as well.
\end{abstract}

\section{Contents}

1. Introduction 2

2. Entropy of endofunctors 4

2.1. Complexity in triangulated categories 4

2.2. Entropy of exact endofunctors

2.3. The case of saturated $A_{\infty}$-categories

2.4. Example: semisimple categories 8

2.5. Regular maps 9

2.6. Entropy of the Serre functor $\quad 10$

2.7. Pseudo-Anosov maps 13

3. Density of phases

3.1. Preliminaries 14

3.2. Dynkin, Euclidean quivers and Kronecker quiver 16

3.3. Kronecker pairs

3.4. Application to quivers 23

3.5. Further examples of Kronecker pairs

4. Open questions 31

4.1. Algebraicity of entropy 31

4.2. Pseudo-Anosov autoequivalences 31

4.3. Birational maps and dynamical spectrum 32

4.4. Complexity and mass 32

4.5. Questions related to Kronecker pairs and density of phases 33

References 


\section{INTRODUCTION}

Recent work of Cantat-Lamy [16] on the Cremona group and Blanc-Cantat [8] on dynamical spectra suggests that there exists a deep parallel between the study of groups of birational automorphisms on one hand, and mapping class groups on the other. Under this parallel, the dynamical degree of a birational map plays the role of the entropy of pseudo-Anosov maps. In the present paper we consider these developments from the perspective of derived categories and their groups of autoequivalences, making direct connections with the classical theory.

In another striking series of papers Gaiotto-Moore-Neitzke [27] and Bridgeland-Smith [15] have established a connection between Teichmüller theory and theory of stability conditions on triangulated categories. An analogy between Teichmüller geodesic flow and the space of stability conditions had been noticed previously in the paper [35] by Soibelman and the fourth author. One of the results is a correspondence between geodesics and stable objects, with slopes of the former giving the phases of the latter. Motivated by this, we study the set of phases of stable objects for general stability conditions, leading to some surprising results.

Let us describe the contents of the paper in more detail. First, we define and study entropy in the context of triangulated and $A_{\infty}$-categories, more specifically dynamical entropy, as a measure of complexity of a dynamical system. This notion comes in a variety of flavors: Let us mention Kolmogorov-Sinai (measure-theoretic) entropy [48, topological entropy [2], and algebraic entropy 6]. In analogy with these notions, we define in Section 2 the entropy of an exact endofunctor of a triangulated category with generator. By taking into account the $\mathbb{Z}$-graded nature of the category one gets not just a single real number, but a function on the real line. In the case of saturated (smooth and proper) $A_{\infty}$-categories, the following foundational results are proven (see Theorems 2.6 and 2.8 for the precise statements)

Theorem. In the saturated case, the entropy of an endofunctor may be computed as a limit of Poincaré polynomials of Ext-groups.

Theorem. In the saturated case (under a certain generic technical condition), there is a lower bound on the entropy given by the logarithm of the spectral radius of the induced action on Hochschild homology.

We compute the entropy of endofunctors in various examples. In the most basic case of semisimple categories the entropy of any endofunctor is described, in log-coordinates, as a branch of a real algebraic curve defined over $\mathbb{Z}$. Next, we show - under a certain generic technical condition that the entropy of a regular endomorphism of a smooth projective variety is given by the logarithm of the spectral radius of the induced map on cohomology. As another direction, we determine the entropy of the Serre functor in a number of cases. The results suggest some relation to the "dimension" of the category. Finally, we show that the entropy of an autoequivalence induced by a pseudo-Anosov map on the Fukaya category of a surface coincides with its topological entropy, the logarithm of the stretch factor. This concludes Section 2 of the paper.

Next, in Section 3, the topological properties of the set of phases of a stability condition on a triangulated category are investigated. This notion of stability was introduced by T. Bridgeland [13] based on a proposal by M. Douglas [21, 22]. It is an essential ingredient in the theory of motivic Donaldson-Thomas invariants developed by Y. Soibelman and the fourth author [35]. As we have mentioned, guided by work of Gaiotto, Moore, and Neitzke [27], Bridgeland and Smith [15] identify spaces of meromorphic quadratic differentials with spaces of stability conditions on 
categories associated with quivers with potential. Moreover, under this correspondence, stable objects are identified with geodesics of finite length. Motivated by the density of the set of slopes of closed geodesics on a Riemann surface we investigate in Section 3 the question whether a given triangulated category admits a stability condition such that the set of phases of stable objects is dense somewhere in the circle.

In case of stability conditions non-dense behavior is possible (Lemma 3.13 and Corollary 3.15):

Theorem. The phases are never dense in an arc for Dynkin and Euclidean quivers ${ }^{1}$

Similarly as in the case of geodesics density property is expected to hold in general.

We introduce the notion of Kronecker pair (Definition 3.23) and show that (Theorem 3.27)

Theorem. If a $\mathbb{C}$-linear triangulated category $\mathcal{T}$ contains a Kronecker pair, s. t. a certain family of stability conditions on it is extendable to the whole category, then the extended stability conditions have phases dense in some arc.

Using this theorem we obtain (Proposition 3.32):

Theorem. Any connected quiver Q, which is neither Euclidean nor Dynkin has a family of stability conditions with phases which are dense in an arc.

We record these findings on density property in the following table:

\begin{tabular}{|l|c|}
\hline Dynkin quivers & $P_{\sigma}$ is always finite \\
\hline Euclidean quivers & $P_{\sigma}$ is either finite or has exactly two limit points \\
\hline All other quivers & $P_{\sigma}$ is dense in an arc for a family of stability conditions \\
\hline
\end{tabular}

where $P_{\sigma}$ denotes the set of stable phases (see Definition 3.4).

By the non-dense behavior of stability conditions we find that on Dynkin and Euclidean quivers the dimensions of Hom spaces of exceptional pairs are strictly smaller than 3 (Corollary 3.31).

Further examples of density of phases (blow ups of projective spaces) are given in subsection 3.5

We summarize the guiding analogies between the classical theory of dynamical systems and the theory of triangulated and $A_{\infty}$-categories in the following table.

\begin{tabular}{|c|c|c|c|c|}
\hline Classical & Shurston & Teodesics \\
Categorical & Stable objects & Stability conditions & Behaviour of \\
\hline
\end{tabular}

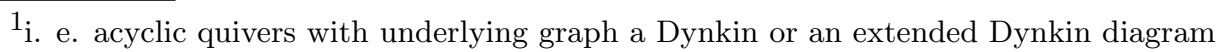


In this paper we only scratch the surface of a rather promising, in our opinion, area of future research. In the final section we suggest some open problems and possible new directions related to stability conditions, birational geometry, and Fukaya categories. First, inspired by the classical theory of pseudo-Anosov maps, we suggest a definition of a pseudo-Anosov functor in the context of triangulated categories with stability conditions. This is conjecturally a direct generalization of the usual notion which has a geometric interpretation in higher dimensions. Furthermore, we propose a connection with the works of Cantat-Lamy [16] and Blanc-Cantat [8]. In [16], based on Gromov's ideas in geometric group theory, the authors prove the nonsimplicity of Cremona group in dimension two. Later, in the paper [8], Blanc and Cantat consider the dynamical spectra of the group of birational transformations as a tool for studying groups of birational automorphisms of surfaces. We propose that this dynamical spectrum is part of a categorical dynamical spectrum. We also briefly explore Gromov's idea that entropy measures growth of volume of submanifolds under the action of a smooth map. From this perspective we conjecture that categorical complexity is related to the mass of objects with respect to a stability condition. Finally, we pose several questions pertaining to the behavior of phases of stable objects.

Acknowledgements: We are grateful to Denis Auroux for many useful discussions. The authors were funded by NSF DMS 0854977 FRG, NSF DMS 0600800, NSF DMS 0652633 FRG, NSF DMS 0854977, NSF DMS 0901330, FWF P 24572 N25, by FWF P20778 and by an ERC Grant.

\section{ENTROPY OF ENDOFUNCTORS}

In this section we define and study the entropy of an exact endofunctor of a triangulated category with generator. Our definition, see Subsection 2.2, is based on a notion of complexity of one object relative to another in such a category discussed in Subsection 2.1. In Subsection 2.3 the case of saturated $A_{\infty}$-categories is treated, for which entropy is more easily computed from dimensions of Ext-groups. The rest of the section is devoted to various examples.

2.1. Complexity in triangulated categories. Suppose $\mathcal{T}$ is a triangulated category with (split-) generator $G$. By definition, this means that for every $E \in \mathcal{T}$ there is an $E^{\prime}$ and a tower of triangles

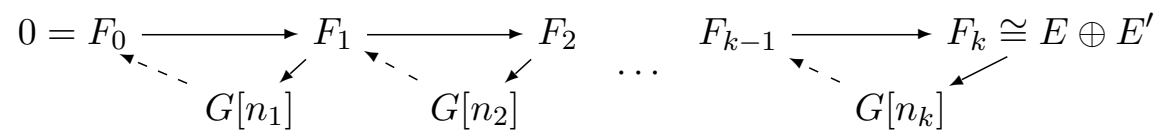

with $k \geq 0, n_{i} \in \mathbb{Z}$. One can ask, for each $E \in \mathcal{T}$, what the least number of shifted copies of $G$ needed is, in order for such a relation to hold. Taking into account the $n_{i}$ 's as well, one arrives at the following definition.

Definition 2.1. Let $E_{1}, E_{2}$ be objects in a triangulated category $\mathcal{T}$. The complexity of $E_{2}$ relative to $E_{1}$ is the function $\delta_{t}\left(E_{1}, E_{2}\right): \mathbb{R} \rightarrow[0, \infty]$ given by

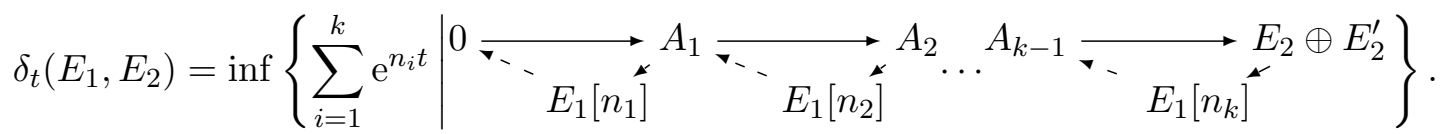

Note that $\delta_{t}\left(E_{1}, E_{2}\right)=+\infty$ iff $E_{2}$ does not lie in the thick triangulated subcategory generated by $E_{1}$. Also, when $\mathcal{T}$ is $\mathbb{Z} / 2$-graded in the sense that $[2] \cong \mathrm{id}_{\mathcal{T}}$, only the value at zero, $\delta_{0}\left(E_{1}, E_{2}\right)$, will be of any use. We collect some basic inequalities in the following proposition. 
Proposition 2.2. Let $\mathcal{T}$ be a triangulated category, $E_{1}, E_{2}, E_{3} \in \mathcal{T}$. Then

(a) (Triangle inequality) $\delta_{t}\left(E_{1}, E_{3}\right) \leq \delta_{t}\left(E_{1}, E_{2}\right) \delta_{t}\left(E_{2}, E_{3}\right)$,

(b) (Subadditivity) $\delta_{t}\left(E_{1}, E_{2} \oplus E_{3}\right) \leq \delta_{t}\left(E_{1}, E_{2}\right)+\delta_{t}\left(E_{1}, E_{3}\right)$,

(c) (Retraction) $\delta_{t}\left(F\left(E_{1}\right), F\left(E_{2}\right)\right) \leq \delta_{t}\left(E_{1}, E_{2}\right)$ for any exact functor $F: \mathcal{T} \rightarrow \mathcal{T}^{\prime}$.

Proof. We will prove only (a). Take $\epsilon>0$ and let

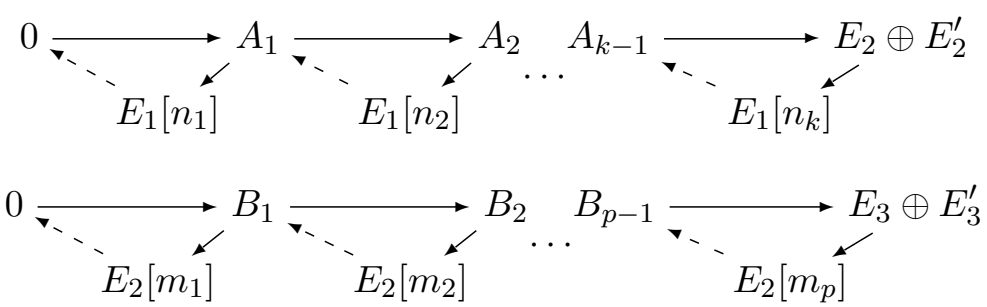

be such that

$$
\sum_{i=1}^{k} \mathrm{e}^{n_{i} t}<\delta_{t}\left(E_{1}, E_{2}\right)+\epsilon, \quad \sum_{j=1}^{p} \mathrm{e}^{m_{j} t}<\delta_{t}\left(E_{2}, E_{3}\right)+\epsilon
$$

Using the given sequences of triangles one can construct a sequence of the type

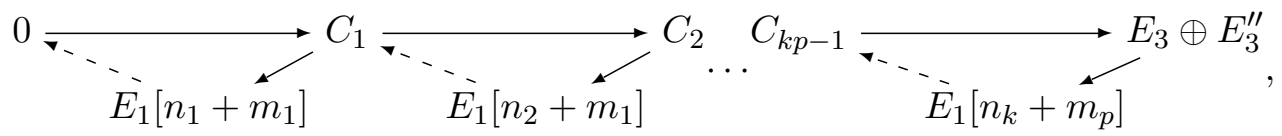

where $E_{3}^{\prime \prime}=E_{3}^{\prime} \oplus E_{2}^{\prime}\left[m_{1}\right] \oplus E_{2}^{\prime}\left[m_{2}\right] \cdots \oplus E_{2}^{\prime}\left[m_{p}\right]$. Hence

$$
\delta_{t}\left(E_{1}, E_{3}\right) \leq \sum_{i, j} \mathrm{e}^{\left(n_{i}+m_{j}\right) t} \leq\left(\delta_{t}\left(E_{1}, E_{2}\right)+\epsilon\right)\left(\delta_{t}\left(E_{2}, E_{3}\right)+\epsilon\right) .
$$

Since this holds for any $\epsilon>0$, the inequality follows.

For complexes of vector spaces the complexity with respect to the ground field is just the Poincaré (Laurent-) polynomial in $\mathrm{e}^{-t}$.

Lemma 2.3. Let $\mathcal{T}=D^{b}(k)$ be the bounded derived category of finite-dimensional vector spaces over a field $k$. Then

$$
\delta_{t}(k, E)=\sum_{n} \operatorname{dim}\left(H^{n} E\right) \mathrm{e}^{-n t}
$$

for any $E \in \mathcal{T}$.

Proof. Any object in $\mathcal{T}$ is isomorphic to its cohomology, which is a direct sum with $\operatorname{dim}\left(H^{n} E\right)$ copies of $k[-n]$ for each $n$. This shows the inequality " $\leq$ ". For the reverse inequality, use the fact that for any exact triangle $E_{1} \rightarrow E_{2} \rightarrow E_{3} \rightarrow E_{1}[1]$ we have $\operatorname{dim} H^{n} E_{2} \leq \operatorname{dim} H^{n} E_{1}+\operatorname{dim} H^{n} E_{3}$.

2.2. Entropy of exact endofunctors. We now come to the main definition of this section. It is based on the observation that if $F$ is an endofunctor of a triangulated category $\mathcal{T}$ with generator $G$, then $\delta_{t}\left(G, F^{n} G\right)$ grows at most exponentially, and the growth constant is independent of the choice of generator. 
Definition 2.4. Let $F: \mathcal{T} \rightarrow \mathcal{T}$ be an exact endofunctor of a triangulated category $\mathcal{T}$ with generator $G$. The entropy of $F$ is the function $h_{t}(F): \mathbb{R} \rightarrow[-\infty,+\infty)$ of $t$ given by

$$
h_{t}(F)=\lim _{n \rightarrow \infty} \frac{1}{n} \log \delta_{t}\left(G, F^{n} G\right) .
$$

The next lemma shows that $h_{t}(F)$ is well-defined.

Lemma 2.5. With $\mathcal{T}, F$ as in the definition, the limit $\lim _{n \rightarrow \infty} \frac{1}{n} \log \left(\delta_{t}\left(G, F^{n} G\right)\right)$ exists in $[-\infty,+\infty)$ for every $t$ and is independent of the choice of generator $G$.

Proof. Using Proposition 2.2 ,

$$
\delta_{t}\left(G, F^{m+n}(G)\right) \leq \delta_{t}\left(G, F^{m}(G)\right) \delta_{t}\left(F^{m}(G), F^{m+n}(G)\right) \leq \delta_{t}\left(G, F^{m}(G)\right) \delta_{t}\left(G, F^{n}(G)\right)
$$

hence existence of the limit follows from Fekete's Lemma: for any subadditive sequence $\left\{a_{n}\right\}_{n \geq 1}$ we have $\lim _{n \rightarrow \infty} \frac{a_{n}}{n}=\inf \left\{\frac{a_{n}}{n} \mid n \geq 1\right\}$. Furthermore, if $G, G^{\prime}$ are generators then

$$
\begin{aligned}
\delta_{t}\left(G^{\prime}, F^{n}\left(G^{\prime}\right)\right) & \leq \delta_{t}\left(G^{\prime}, G\right) \delta_{t}\left(G, F^{n}(G)\right) \delta_{t}\left(F^{n}(G), F^{n}\left(G^{\prime}\right)\right) \\
& \leq \delta_{t}\left(G^{\prime}, G\right) \delta_{t}\left(G, G^{\prime}\right) \delta_{t}\left(G, F^{n}(G)\right)
\end{aligned}
$$

which implies the second claim.

We conclude this subsection with some remarks on the behaviour of $h_{t}$ with respect to composition of functors. Clearly, $h_{t}$ is constant on conjugacy classes, and $h_{t}\left(F^{n}\right)=n h_{t}(F)$ for $n \geq 1$. If $\mathcal{T} \neq 0$, then $h_{0}\left(\mathrm{id}_{\mathcal{T}}\right)=0$, but this may fail for other values of $t$. In general, nothing can be said about $h_{t}(F \circ H)$ from $h_{t}(F)$ and $h_{t}(H)$ alone, but if $F$ and $H$ commute, then $h_{t}(F \circ H) \leq h_{t}(F)+h_{t}(H)$.

2.3. The case of saturated $A_{\infty}$-categories. For background on $A_{\infty}$-categories see for example [37] and [34. We will work over a fixed field $k$. An $A_{\infty}$-category $\mathcal{C}$ is triangulated if every compact object in $\operatorname{Mod}(\mathcal{C})$ is quasi-representable. The homotopy category, $H^{0} \mathcal{C}$, of a triangulated $A_{\infty^{-}}$ category is triangulated in the sense of Verdier. An $A_{\infty}$-category is saturated if it is triangulated and Morita-equivalent to a smooth and compact $A_{\infty}$-algebra $A$.

In the case of saturated $A_{\infty}$-categories one can, for the purpose of computing the entropy, replace $\delta_{t}(A, B)$ with the Poincaré polynomial of $\operatorname{Ext}^{*}(A, B)$ in $\mathrm{e}^{-t}$.

Theorem 2.6. Let $\mathcal{C}$ be a saturated $A_{\infty}$-category and $F$ an endofunctor of $\mathcal{C}$. Then

$$
h_{t}(F)=\lim _{N \rightarrow \infty} \frac{1}{N} \log \sum_{n \in \mathbb{Z}} \operatorname{dim} \operatorname{Ext}^{n}\left(G, F^{N} G\right) \mathrm{e}^{-n t}
$$

for any generator $G$ of $\mathcal{C}$.

Proof. Note first that

$$
\delta_{t}\left(k, R \operatorname{Hom}\left(G, F^{N} G\right)\right)=\sum_{n} \operatorname{dim} \operatorname{Ext}^{n}\left(G, F^{N} G\right) \mathrm{e}^{-n t}
$$

by Lemma 2.3. We will show that there exist $C_{1}, C_{2}: \mathbb{R} \rightarrow \mathbb{R}_{>0}$, depending on $G$, such that

$$
C_{1}(t) \delta_{t}(G, E) \leq \delta_{t}(k, R \operatorname{Hom}(G, E)) \leq C_{2}(t) \delta_{t}(G, E)
$$

for every $E \in \mathcal{C}$. The theorem follows from $(9)$ by setting $E=F^{N} G$, taking the logarithm, dividing by $N$, and passing to the limit $N \rightarrow \infty$. 
For the second inequality, apply Proposition 2.2 to the functor $R \operatorname{Hom}\left(G,{ }_{-}\right): \mathcal{C} \rightarrow D^{b}(k)$, which is well defined by local properness of $\mathcal{C}$. We get

$$
\begin{aligned}
\delta_{t}(k, R \operatorname{Hom}(G, E)) & \leq \delta_{t}(k, R \operatorname{Hom}(G, G)) \delta_{t}(R \operatorname{Hom}(G, G), R \operatorname{Hom}(G, E)) \\
& \leq \delta_{t}(k, R \operatorname{Hom}(G, G)) \delta_{t}(G, E)
\end{aligned}
$$

where the first factor is a function $C_{2}(t): \mathbb{R} \rightarrow \mathbb{R}_{>0}$ independent of $E$. Note that we did not use the assumption that $\mathcal{C}$ is smooth.

On the other hand

$$
\begin{aligned}
\delta_{t}(G, E) & \leq \delta_{t}(G, G \otimes R \operatorname{Hom}(G, E)) \delta_{t}(G \otimes R \operatorname{Hom}(G, E), E) \\
& \leq \delta_{t}(k, R \operatorname{Hom}(G, E)) \delta_{t}\left(G \otimes R \operatorname{Hom}\left(G,{ }_{-}\right), \operatorname{id}_{\mathcal{C}}\right)
\end{aligned}
$$

by the retraction map property of the functors $G \otimes_{-}: D^{b}(k) \rightarrow \mathcal{C}$ and $\Phi \mapsto \Phi(E):$ Fun $(\mathcal{C}, \mathcal{C}) \rightarrow \mathcal{C}$. It remains to show that the second factor, $\delta_{t}\left(G \otimes R \operatorname{Hom}\left(G,{ }_{-}\right), \mathrm{id}_{\mathcal{C}}\right)$ is in fact finite, i.e. that id lies in the subcategory of $\operatorname{Fun}(\mathcal{C}, \mathcal{C})$ generated by $G \otimes R \operatorname{Hom}\left(G,{ }_{-}\right)$. But this is just equivalent to smoothness of $\mathcal{C}$. Indeed, let $A=\operatorname{End}(G)$, then the functor $G \otimes R \operatorname{Hom}\left(G,{ }_{-}\right)$corresponds to the bimodule $A \otimes_{k} A^{\text {op }}$ and Ide corresponds to the diagonal bimodule $A$. This completes the proof of (9) and the theorem.

Suppose that $\mathcal{C}$ is a saturated $A_{\infty}$-category over $\mathbb{C}$. Since $\mathcal{C}$ is smooth and proper, its Hochschild homology, $H H_{*}(\mathcal{C})$, is finite-dimensional. Any endofunctor $F$ of $\mathcal{C}$ induces a linear map on $H H_{*}(\mathcal{C})$ and we may consider the logarithm of its spectral radius as a kind of "homological entropy". We will show that, under a certain generic condition discussed in the lemma below, this number is bounded above by $h_{0}(F)$.

Lemma 2.7. Let $V$ be a finite-dimensional $\mathbb{Z} /(2)$-graded $\mathbb{C}$-vector space, $A=A_{+} \oplus A_{-}$a homogeneous endomorphism of $V$. Suppose that $A_{+}, A_{-}$do not have the same eigenvalues, with multiplicity, on $\{|\lambda|=\rho(A)\}$. Then

$$
\limsup _{n \rightarrow \infty}\left|\mathrm{s} \operatorname{Tr} A^{n}\right|^{1 / n}=\rho(A)
$$

Proof. We may assume that $\rho(A) \neq 0$. Let $\alpha_{1}, \ldots, \alpha_{m}$ be the eigenvalues of $A_{+}$and $\beta_{1}, \ldots, \beta_{n}$ those of $A_{-}$. Consider the identity

$$
\sum_{n=0}^{\infty} \mathrm{s} \operatorname{Tr} A^{n} z^{n}=\sum_{k=1}^{m} \frac{1}{1-\alpha_{k} z}-\sum_{l=1}^{n} \frac{1}{1-\beta_{l} z}
$$

which holds for $|z|$ sufficiently small. If $R$ denotes the radius of convergence of the series on the left hand side of (14), then

$$
R^{-1}=\limsup _{n \rightarrow \infty}\left|\mathrm{s} \operatorname{Tr} A^{n}\right|^{1 / n} .
$$

On the other hand, it follows from the conditions on $A$ that $(14)$, extended meromorphically to $\mathbb{C}$, has a pole on $\{|z|=1 / \rho(A)\}$, and no poles closer to the origin. Hence $R=\rho(A)$, and the proof of the lemma is complete.

Theorem 2.8. Let $\mathcal{C}$ be a saturated $A_{\infty}$-category over $\mathbb{C}$ and $F: \mathcal{C} \rightarrow \mathcal{C}$ a functor. Assume that the induced map on Hochschild homology, $H_{*}(F)$, satisfies the condition of Lemma 2.7, then

$$
\log \rho\left(H H_{*}(F)\right) \leq h_{0}(F) .
$$


Proof. Let $S$ denote the Serre functor of C. By Lemma 2.3 ,

$$
\delta_{0}\left(k, R \operatorname{Hom}\left(F^{N}, S\right)\right)=\sum_{n} \operatorname{dim} \operatorname{Ext}^{n}\left(F^{N}, S\right)
$$

thus

$$
\left|\chi\left(R \operatorname{Hom}\left(F^{N}, S\right)\right)\right| \leq \delta_{0}\left(k, R \operatorname{Hom}\left(F^{N}, S\right)\right) .
$$

Applying the triangle inequality,

$$
\begin{aligned}
\delta_{0}(k, & \left.R \operatorname{Hom}\left(F^{N}, S\right)\right) \\
\leq & \delta_{0}\left(k, R \operatorname{Hom}\left(G \otimes R \operatorname{Hom}\left(G,{ }_{-}\right), S\right)\right) \delta_{0}\left(R \operatorname{Hom}\left(G \otimes R \operatorname{Hom}\left(G,{ }_{-}\right), S\right), R \operatorname{Hom}\left(F^{N}, S\right)\right) .
\end{aligned}
$$

The first factor is independent of $N$, while the second is bounded above by $\delta_{0}\left(G \otimes R \operatorname{Hom}\left(G,{ }_{-}\right), F^{N}\right)$ as follows from the retraction map property of the functor $R \operatorname{Hom}\left({ }_{-}, S\right): \operatorname{Fun}(\mathrm{C}, \mathrm{e}) \rightarrow D^{b}(k-\operatorname{Vect})^{\mathrm{op}}$. Now,

$$
\begin{aligned}
\delta_{0}(G \otimes & \left.R \operatorname{Hom}\left(G,_{-}\right), F^{N}\right) \\
\leq & \delta_{0}\left(G \otimes R \operatorname{Hom}\left(G,,_{-}\right), F^{N} \circ\left(G \otimes R \operatorname{Hom}\left(G,,_{-}\right)\right)\right) \delta_{0}\left(F^{N} \circ\left(G \otimes R \operatorname{Hom}\left(G,,_{-}\right)\right), F^{N}\right) .
\end{aligned}
$$

By the retraction property of $F^{N} \circ_{-}$, the second factor is bounded above by $\delta_{0}\left(G \otimes R \operatorname{Hom}\left(G,{ }_{-}\right), \operatorname{id}_{\mathcal{C}}\right)$ which is independent of $N$ and finite by smoothness of $\mathcal{C}$. The first factor is bounded above by $\delta_{0}\left(G, F^{N} G\right)$, hence

$$
\limsup _{N \rightarrow \infty}\left|\chi\left(R \operatorname{Hom}\left(F^{N}, S\right)\right)\right|^{1 / N} \leq \mathrm{e}^{h_{0}(F)} .
$$

By the Lefschetz fixed point theorem for Hochschild homology [38] we have

$$
\chi\left(R \operatorname{Hom}\left(F^{N}, S\right)\right)=\operatorname{sTr}\left(H H_{*}\left(F^{N}\right)\right)
$$

and by Lemma 2.7 ,

$$
\limsup _{N \rightarrow \infty}\left|\mathrm{s} \operatorname{Tr}\left(H H_{*}(F)^{N}\right)\right|^{1 / N}=\rho\left(H H_{*}(F)\right)
$$

which completes the proof.

Remark 2.9. We suspect that the conclusion of Theorem 2.8 holds without the condition on $F$.

2.4. Example: semisimple categories. Fix a finite set $X$ and a field $k$. Let $D^{b}(X)$ denote the triangulated dg-category of $X$-indexed families of bounded complexes over $k$. Any (additive, graded) endofunctor of $D^{b}(X)$ is isomorphic to one of the form

$$
\Phi_{K}(V)=\left(p_{1}\right)_{*}\left(K \otimes p_{2}^{*} V\right)
$$

for some $K \in D^{b}(X \times X)$ with trivial differential. Such a $K$ corresponds to a matrix of Poincaré (Laurent-) polynomials

$$
P_{K}(z)=\left(\sum_{n \in \mathbb{Z}} \operatorname{dim}\left(K_{x, y}^{n}\right) z^{n}\right)_{x, y \in X}
$$

with non-negative integer coefficients. Note that composition of functors corresponds to multiplication of matrices. 
Let $G \in D^{b}(X)$ be the generator with $G_{x}=k$ for $x \in X$. Then

$$
\sum_{n \in \mathbb{Z}} \operatorname{dim} \operatorname{Ext}^{n}\left(G, \Phi_{K}^{N}(G)\right) e^{-n t}=\left\|P_{K}^{N}\left(e^{-t}\right)\right\|_{1}
$$

where $\|A\|_{1}=\sum\left|a_{i j}\right|$ is the matrix 1-norm and we use non-negativity of $P_{K}^{N}\left(e^{-t}\right)$. We obtain

$$
h_{t}\left(\Phi_{K}\right)=\log \rho\left(P_{K}\left(e^{-t}\right)\right)
$$

by Gelfand's formula for the spectral radius

$$
\rho(A)=\lim _{n \rightarrow \infty}\left\|A^{n}\right\|^{\frac{1}{n}}
$$

which holds for any matrix norm.

We can say a bit more about the function $\rho\left(P_{K}(x)\right)$. By Perron-Frobenius theory, the spectral radius of a non-negative matrix is an eigenvalue. Hence, if we consider the spectral curve

$$
C=\left\{(x, \lambda) \in \mathbb{R}_{>0} \times \mathbb{R} \mid \operatorname{det}\left(P_{K}(x)-\lambda\right)=0\right\}
$$

then the graph of $\rho\left(P_{K}(x)\right): \mathbb{R}_{>0} \rightarrow \mathbb{R}$ is the maximal branch of $C$.

2.5. Regular maps. Let $X$ be a smooth projective variety over $\mathbb{C}, f: X \rightarrow X$ a regular map, and $f^{*}: D^{b}(X) \rightarrow D^{b}(X)$ the pullback functor. We will show that, under the generic condition of Lemma 2.7, the entropy of $f^{*}$ is constant and equal to the logarithm of the spectral radius of $H^{*}(f ; \mathbb{Q})$, the induced map on cohomology. By a result of S. Friedland $[26], \log \rho\left(H^{*}(f ; \mathbb{Q})\right)$ is also equal to the topological entropy of $f$, more generally for compact Kähler manifolds.

We begin with a lemma which gives a sufficient condition for the entropy $h_{t}(F)$ to be constant in $t$.

Lemma 2.10. Let $\mathcal{C}$ be a saturated $A_{\infty}$-category and $F$ an endofunctor of $\mathcal{C}$. Suppose there exists a generator $G$ of $\mathcal{C}$ and $M \geq 0$ such that

$$
\operatorname{Ext}^{n}\left(G, F^{N} G\right)=0 \quad \text { for }|n|>M, N \geq 0
$$

then $h_{t}(F)$ is a constant function.

Proof. Use

$$
\operatorname{dim} \operatorname{Ext}^{n}\left(G, F^{N} G\right) e^{-M|t|} \leq \operatorname{dim} \operatorname{Ext}^{n}\left(G, F^{N} G\right) e^{-n t} \leq \operatorname{dim} \operatorname{Ext}^{n}\left(G, F^{N} G\right) e^{M|t|}
$$

to conclude $h_{0}(F) \leq h_{t}(F) \leq h_{0}(F)$.

Note that Lemma 2.10 applies in particular to functors preserving a bounded t-structure of finite homological dimension, as the generator can be chosen to lie in the heart of the t-structure.

Theorem 2.11. Let $X$ be a smooth projective variety over $\mathbb{C}, f: X \rightarrow X$ a regular map, and $f^{*}: D^{b}(X) \rightarrow D^{b}(X)$ the pullback functor. Suppose that the induced map on cohomology, $H^{*}(f ; \mathbb{Q})$, satisfies the condition of Lemma 2.7. Then $h_{t}\left(f^{*}\right)$ is constant and equal to $\log \rho\left(H^{*}(f ; \mathbb{Q})\right)$.

Proof. Clearly, $f^{*}$ preserves $\operatorname{coh}(X) \subset D^{b}(X)$ which has finite homological dimension, since $X$ is smooth and proper. Hence, by Lemma 2.10, $h_{t}\left(f^{*}\right)$ is constant in $t$.

We will show that

$$
h_{0}\left(f^{*}\right) \leq \log \rho\left(H^{*}(f, \mathbb{Q})\right)
$$


without the assumption of Lemma 2.7. The claim then follows from Theorem 2.8 by the HochschildKostant-Rosenberg isomorphism and Hodge theory which give

$$
H H_{*}\left(D^{b}(X)\right) \cong H^{*}(X ; \mathbb{C})
$$

as $\mathbb{Z} / 2$-graded vector spaces.

It remains to show $(30)$. Choose $L \in \operatorname{Pic}(X)$ very ample and consider

$$
G=\bigoplus_{k=1}^{d+1} L^{k}
$$

where $d=\operatorname{dim} X$. Both $G$ and $G^{*}$ are generators of $D^{b}(X)$ by [41. Since $X$ is projective, $f$ preserves the nef cone in $N^{1}(X)_{\mathbb{R}}$. In particular, $\left(f^{*}\right)^{N} G^{*}$ is a sum of anti-nef line bundles for every $N \geq 0$. Note that $G^{*}$ itself is a sum of anti-ample line bundles, hence, using nef + ample $=$ ample, we conclude that

$$
G^{*} \otimes\left(f^{*}\right)^{N} G^{*}=\bigoplus_{1 \leq k, l \leq d+1} L^{-k} \otimes\left(f^{*}\right)^{N} L^{-l}
$$

is a sum of anti-ample line bundles. Thus, by the Kodaira vanishing theorem, $\operatorname{Ext}^{*}\left(G,\left(f^{*}\right)^{N} G^{*}\right)$ is concentrated in degree $d$. We get

$$
\begin{aligned}
\operatorname{dim} \operatorname{Ext}^{*}\left(G,\left(f^{*}\right)^{N} G^{*}\right) & =\left|\chi \operatorname{Ext}^{*}\left(G,\left(f^{*}\right)^{N} G^{*}\right)\right| \\
& =\left|\int \operatorname{ch}\left(G^{*}\right)\left(f^{*}\right)^{N} \operatorname{ch}\left(G^{*}\right) \operatorname{td}(X)\right|
\end{aligned}
$$

where $f^{*}$ is the induced map on $H^{*}(X ; \mathbb{Q})$. It follows that

$$
\lim _{N \rightarrow \infty} \sqrt[N]{\operatorname{dim} \operatorname{Ext}^{*}\left(G,\left(f^{*}\right)^{N} G^{*}\right)} \leq \rho\left(H^{*}(X ; \mathbb{Q})\right)
$$

which shows the claim, since the left hand side is $\exp \left(h_{0}\left(f^{*}\right)\right)$ by Theorem 2.6. More precisely, we use here a variant of this theorem, with the same proof, where one is allowed to take two different generators.

2.6. Entropy of the Serre functor. The notion of a Serre functor was introduced by Bondal and Kapranov in [7]. If it exists, it is unique up to natural isomorphism, and thus its entropy an invariant of the triangulated category. In the examples below, the entropy of the Serre functor will always turn out to be of the linear form $a t+b$, with $a$ having some interpretation as a dimension. We note however, that this can fail in general, for example for categories which are orthogonal sums.

2.6.1. Fractional Calabi-Yau categories. A triangulated category $\mathcal{T}$ with Serre functor $S$ is called fractional Calabi-Yau of dimension $\frac{m}{n} \in \mathbb{Q}$ if

$$
S^{n} \cong[m]
$$

c.f. [31]. Suppose that $\mathcal{T}$ is the homotopy category of a saturated $A_{\infty}$-category $\mathcal{C}$. Using

$$
h_{t}\left(F^{n}\right)=n h_{t}(F) \quad \text { for } n \geq 1, \quad h_{t}([n])=n t \quad \text { for } n \in \mathbb{Z}
$$

we see that

$$
h_{t}(S)=\frac{m}{n} t
$$


in this case.

2.6.2. Smooth projective varieties. Let $X$ be a smooth projective variety over a field $k$. The bounded derived category of coherent sheaves on $X, D^{b}(X)=D^{b}(\operatorname{coh}(X))$ is saturated with Serre functor

$$
S={ }_{-} \otimes \omega_{X}[\operatorname{dim} X]
$$

where $\omega_{X}$ is the canonical sheaf.

Proposition 2.12. $h_{t}(S)=\operatorname{dim}(X) t$

Note that in particular, if $\mathcal{C}$ is a saturated category and $h_{t}(S)$ is not of the form $n t$ for some $n \in \mathbb{Z}_{\geq 0}$, then $\mathcal{C}$ cannot come from an algebraic variety.

Proof. This is equivalent to $h_{t}\left(-\otimes \omega_{X}\right)=0$, which is a special case of Lemma 2.13 below.

Lemma 2.13. Let $X$ be a smooth projective variety over a field $k, L \in \operatorname{Pic}(X)$. Then $h_{t}(-\otimes L)=0$.

Proof. By lemma 2.10, $h_{t}(-\otimes L)$ is constant. Let $G \in \operatorname{coh}(X)$ be a generator of $D^{b}(X)$. It suffices to show that

$$
\sum_{n \in \mathbb{Z}} \operatorname{dim} \operatorname{Ext}^{n}\left(G, G \otimes L^{N}\right)=O\left(N^{d}\right), \quad N \rightarrow \infty
$$

for some $d$. This holds by the standard fact that

$$
\operatorname{dim} H^{n}\left(\mathcal{O}_{X}, \mathcal{F} \otimes L^{N}\right)=O\left(N^{\operatorname{dim} X}\right), \quad N \rightarrow \infty
$$

for any coherent sheaf $\mathcal{F}, n \geq 0$, see [36].

2.6.3. Quivers. Let $Q$ be a quiver. We assume throughout that $Q$ is connected and does not have oriented cycles. Fix a field $k$ and form the path algebra $A=k Q$ of $Q$. Let $\mathcal{A}$ be the category of finite dimensional left modules over $A$. Its bounded derived category, $D^{b} \mathcal{A}$, is saturated with Serre functor isomorphic to the derived functor of $\operatorname{Hom}(-, A)^{*}$.

Lemma 2.14. If $X \in \mathcal{A}$ is projective, then $S(X) \in \mathcal{A}$ and $S(X)$ is injective. If $X$ has no projective summands, then $S(X) \in \mathcal{A}[1]$.

Proof. Note first that $\mathcal{A}$ is hereditary and thus any indecomposable object in $D^{b} \mathcal{A}$ is contained in $\mathcal{A}[n]$ for some $n \in \mathbb{Z}$. Furthermore, if $M \in \mathcal{A}$ is indecomposable, then $S(M)$ is contained either in $\mathcal{A}$ or in $\mathcal{A}[1]$. Suppose $P$ is projective, then

$$
\operatorname{Ext}^{n}(P, M)=\operatorname{Ext}^{-n}(M, S(P))=0 \quad \text { for } n \neq 0, M \in \mathcal{A}
$$

hence $S(P) \in \mathcal{A}$ and $S(P)$ is injective. If $N \in \mathcal{A}$ is indecomposable and not projective, then there exists an $M \in \mathcal{A}$ with

$$
\operatorname{Ext}^{1}(N, M)=\operatorname{Ext}^{-1}(M, S(N)) \neq 0
$$

thus $S(N) \notin \mathcal{A}$.

Let $e_{i}$ be the idempotent corresponding to the vertex $i \in Q_{0}$. Recall that $A e_{i}, i \in Q_{0}$ is a complete list of indecomposable projectives and $\left(e_{i} A\right)^{*}, i \in Q_{0}$ is a complete list of indecomposable injectives.

Lemma 2.15. If $Q$ is not Dynkin, then $\Phi^{-N}(P) \in \mathcal{A}$ for any projective module $P$ and $N \geq 0$. 
Proof. By the previous lemma, $\Phi^{-1}(M) \in \mathcal{A}$ for an indecomposable $M \in \mathcal{A}$ as long as $M$ is not injective. Suppose that $P$ is projective and $M=\Phi^{-N}(P)$ is injective. Then $M$ is both preprojective and preinjective, thus $A$ of finite representation type by a result of [3] , contradicting the assumption that $Q$ is not Dynkin.

If $Q$ is Dynkin, then $D^{b} \mathcal{A}$ is fractional Calabi-Yau, see [31. We assume from now on that $Q$ is not Dynkin. Then, by the lemma

$$
\operatorname{Ext}^{n}\left(A, \Phi^{-N}(A)\right)=0 \quad \text { for } N \geq 0, n \neq 0
$$

and using $\operatorname{dim} \operatorname{Hom}(A, M)=\operatorname{dim} M$ we get

$$
h_{t}\left(\Phi^{-1}\right)=\lim _{N \rightarrow \infty} \frac{1}{N} \log \operatorname{dim} \Phi^{-N} A .
$$

Furthermore, by duality,

$$
\operatorname{dim} \operatorname{Ext}^{n}\left(A, \Phi^{N} A\right)=\operatorname{dim} \operatorname{Ext}^{1-n}\left(A,\left(\Phi^{-1}\right)^{N-1} A\right)
$$

hence

$$
h_{t}(S)=h_{t}(\Phi)+t=h_{t}\left(\Phi^{-1}\right)+t .
$$

At this point, the problem of computing $h_{t}\left(\Phi^{-1}\right)$ is one of linear algebra, since $\operatorname{dim} M$ depends only on

$$
M \in K_{0}(\mathcal{A}) \cong \mathbb{Z}^{Q_{0}}
$$

where a natural basis of $K_{0}(\mathcal{A})$ is given by the simple modules. Recall that the Euler form

$$
\langle M, N\rangle=\sum_{n \in \mathbb{Z}}(-1)^{n} \operatorname{dim} \operatorname{Ext}^{n}(M, N)
$$

is a bilinear form on $K_{0}(\mathcal{A})$ with matrix $E$ given by

$$
E_{i j}=\delta_{i j}-n_{i j}
$$

where $n_{i j}$ is the number of arrows from the $j$-th to the $i$-th vertex. Let $[S]$ denote the induced map on $K_{0}(\mathcal{A})$. By the defining property of the Serre functor, $E^{\top}=E[S]$, hence

$$
[S]=E^{-1} E^{\top}, \quad[\Phi]=-E^{-1} E^{\top}, \quad\left[\Phi^{-1}\right]=-E^{-\top} E .
$$

The linear map $[\Phi]$ is classically the Coxeter transformations for the Cartan matrix $E+E^{\top}$. Its spectral properties were investigated by Dlab and Ringel in [23]. They find that $\rho=\rho([\Phi])=$ $\rho\left(\left[\Phi^{-1}\right]\right)$ is an eigenvalue of $[\Phi], \rho \geq 1$, and $\rho=1$ if and only if $Q$ is of extended Dynkin type. Furthermore, if $P$ is projective, then

$$
\lim _{N \rightarrow \infty}\left(\operatorname{dim} \Phi^{-N} P\right)^{\frac{1}{N}}=\rho .
$$

We summarize our results.

Theorem 2.16. Let $Q$ be a connected quiver without oriented cycles, not of Dynkin type, and $S$ the Serre functor on $D^{b}(k Q)$. Then

$$
h_{t}(S)=t+\log \rho([S])
$$

and $\rho([S]) \geq 1$ with equality if and only if $Q$ is of extended Dynkin type. 
2.7. Pseudo-Anosov maps. A great insight of Thurston was that a "typical" element of the mapping class group of a surface $M$ can be represented by a pseudo-Anosov map [49, 25, which is by definition a homeomorphisms $\phi: M \rightarrow M$ such that there exist transverse measured foliations $\left(\mathcal{F}^{s}, \mu^{s}\right),\left(\mathcal{F}^{u}, \mu^{u}\right)$ and $\lambda>1$, such that

$$
\phi\left(\mathcal{F}^{s}, \mu^{s}\right)=\left(\mathcal{F}^{s}, \frac{1}{\lambda} \mu^{s}\right), \quad \phi\left(\mathcal{F}^{u}, \mu^{u}\right)=\left(\mathcal{F}^{u}, \lambda \mu^{u}\right) .
$$

The stretch factor $\lambda$ can be computed as follows. Let $\mathcal{S}$ be the set of isotopy classes of simple closed curves on $M$ which do not contract to a point. For $\alpha, \beta \in \mathcal{S}$ the geometric intersection number $i(\alpha, \beta)$ is the minimum number of intersection points of simple closed curves representing $\alpha$ and $\beta$. Then for $\phi, \lambda$ as above and $\alpha, \beta \in \mathcal{S}$ one has

$$
\lim _{n \rightarrow \infty} \sqrt[n]{i\left(\alpha, \phi^{n}(\beta)\right)}=\lambda
$$

We restrict to the case when $M$ is closed, orientable, and of genus $g>1$. The Fukaya category $\operatorname{Fuk}(M)$ is a $\mathbb{Z} / 2$-graded $A_{\infty}$-category over the Novikov field $\Lambda_{\mathbb{R}}$. For our purposes, objects are embedded curves with orientation and the bounding spin structure. Let $D^{\pi} \operatorname{Fuk}(M)$ denote the triangulated hull, which is locally proper (in the $\mathbb{Z} / 2$-graded sense), and has a generator given by a collection of loops [47, 24]. In fact, an explicit resolution of the diagonal is constructed in [47, 24], proving homological smoothness. Thus $D^{\pi} \operatorname{Fuk}(M)$ is saturated. An element $\phi$ of the mapping class group of $M$ induces an autoequivalence $\phi_{*}$ of $D^{\pi} \operatorname{Fuk}(M)$.

Theorem 2.17. Let $\phi$ be a pseudo-Anosov map with stretch factor $\lambda$, then $h_{0}\left(\phi_{*}\right)=\log \lambda$.

It is a classical result, see [25], that the topological entropy of a pseudo-Anosov map $\phi$ is $\log \lambda$, and that $\phi$ minimizes topological entropy in its isotopy class.

Proof. Note first that Theorem 2.6 is also valid, with essentially the same proof, for saturated $\mathbb{Z} / 2$-graded $A_{\infty}$-categories in the sense that

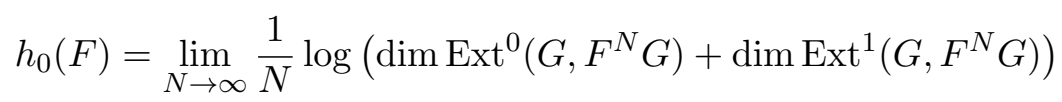

for any endofunctor $F$. On the other hand, if $\alpha, \beta$ are simple closed loops in $M$ then

$$
\operatorname{dim} \operatorname{Ext}^{0}\left(\alpha, \phi^{N}(\beta)\right)+\operatorname{dim} \operatorname{Ext}^{1}\left(\alpha, \phi^{N}(\beta)\right)=i\left(\alpha, \phi^{N}(\beta)\right)
$$

for $N \gg 0$ by Lemma 2.18 below. Now, $D^{\pi} \operatorname{Fuk}(M)$ has a generator $G$ which is a direct sum of $2 g+1$ simple loops. In view of 56 the theorem follows.

The following is considered well-known to experts in Floer theory.

Lemma 2.18. Let $M$ be a closed surface of positive genus with symplectic structure, $\alpha$ and $\beta$ simple closed loops on $M$ which are not isotopic. Then

$$
\operatorname{dim} H F^{0}(\alpha, \beta)+\operatorname{dim} H F^{1}(\alpha, \beta)=i(\alpha, \beta)
$$

where $H F^{k}(\alpha, \beta)$ denotes Floer cohomology over the Novikov field.

Proof. If $\alpha$ and $\beta$ are already in minimal position, i.e. $i(\alpha, \beta)=|\alpha \cap \beta|$, then (59) is clear, since there are no immersed bi-gons with boundary on $\alpha \cup \beta$, and thus the Floer differential vanishes on $C F^{*}(\alpha, \beta)$ which has a basis given by the intersection points.

In general, there is an embedded loop $\alpha^{\prime}$ which is isotopic to $\alpha$ and intersects $\beta$ minimally. Moreover, we can construct an isotopy from $\alpha$ to $\alpha^{\prime}$ which is a composition of Hamiltonian isotopies 
and isotopies which do not create or remove any intersection points between $\alpha$ and $\beta$. The invariance of $H F^{*}(\alpha, \beta)$ under the former is true by the general theory, we will show that invariance also holds for the latter.

Let us observe first that near each intersection point $p$ of $\alpha$ and $\beta$ there is a chart taking $\alpha$ to the $\mathrm{x}$-axis and $\beta$ to the $\mathrm{y}$-axis. In such a chart, any bi-gon contributing to $d[p]$ must map locally to either the second or fourth quadrant, see [1. Furthermore, the assumption on $\alpha$ and $\beta$ ensures that bi-gons contributing to $d^{0}$ (resp. $d^{1}$ ) cannot form a closed chain.

Let $M$ be the matrix representing the Floer differential $d^{0}$ or $d^{1}$, with respect to the basis given by intersection points. Since $\alpha$ and $\beta$ are not isotopic, there can be at most one bi-gon contributing to any entry of $M$, hence the position of the non-zero entries does not change under the isotopy. The observations made above translate into the following properties of $M$.

(1) Any column or row of $M$ has at most two non-zero entries.

(2) Let $Q$ be the quiver with vertices the columns and rows of $M$ and an arrow from $i$ to $j$ if the entry in the $i$-th column and $j$-th row of $M$ is non-zero. Then the underlying undirected graph of $Q$ has no loops.

One shows that the rank of a matrix satisfying the second property is determined by the positions of the non-zero entries alone, and not the specific values. We conclude that $\operatorname{dim} H F^{*}(\alpha, \beta)$ is invariant under isotopy.

\section{Density of Phases}

\subsection{Preliminaries.}

3.1.1. On Bridgeland stability conditions. We start by recalling some definitions and results by Bridgeland. Here we introduce the notations $\mathbb{H}^{\mathcal{A}}$ (see Definition 3.2 and Remark 3.3) and $P_{\sigma}$ (the set of semistable phases of a stability condition $\sigma$ ), which are useful later.

In [13] a stability conditions on a triangulated category $\mathcal{T}$ is defined as a pair $\sigma=(\mathcal{P}, Z)$ satisfying certain axioms, where the first component is a family of full additive subcategories $\{\mathcal{P}(t)\}_{t \in \mathbb{R}}$ (by the axioms it follows that they are abelian) and the second component is a group homomorphism

$K_{0}(\mathcal{T}) \stackrel{Z}{\longrightarrow} \mathbb{C}$. The non-zero objects in $\mathcal{P}(t)$ are said to be $\sigma$-semistable of phase $t$ and the phase $\phi_{\sigma}(E)$ of a semistable $E \in \mathcal{P}(t)$ is well defined by $\phi_{\sigma}(E)=t$.

To give a description of the Bridgeland stability conditions on $\mathcal{T}$, we recall first the following definition:

Definition 3.1. Let $\left(\mathcal{A}, K_{0}(\mathcal{A}) \stackrel{Z}{\longrightarrow} \mathbb{C}\right)$ be an abelian category and a stability function on it $2^{2}$. $A$ non-zero object $X \in \mathcal{A}$ is said to be $Z$-semistable of phase $t$ if every $\mathcal{A}$-monic $X^{\prime} \rightarrow X$ satisfies ${ }^{3}$ $\arg Z\left(X^{\prime}\right) \leq \arg Z(X)=\pi t$ (if equality is attained only for $X^{\prime} \cong X$ then $X$ is said to be stable).

If $\mathcal{A} \subset \mathcal{T}$ is a bounded $t$-structure and $Z: K_{0}(\mathcal{A}) \rightarrow \mathbb{C}$ is a stability function, which satisfies the HN property ([13, Definition 2.3]) then the pair $\sigma=\left(\mathcal{P}, Z_{e}\right)$ defined by(see the proof of [13, Proposition 5.3]):

a) If $t \in(0,1]$, define $\mathcal{P}(t) \subset \mathcal{A}$ as the set of $Z$-semistable objects of phase $t$ in $\mathcal{A}$ (as defined in definition 3.1). If $t \in(n, n+1], n \in \mathbb{Z}$, define $\mathcal{P}(t)=T^{n}(\mathcal{P}(t-n))$.

\footnotetext{
2.e. $Z$ is homomorphism, s. t. $Z(X) \in \mathbb{H}=\{r \exp (\mathrm{i} \pi t) \mid r>0$ and $0<t \leq 1\}$ for $X \in \mathcal{A}, X \neq 0$

${ }^{3}$ For $u \in \mathbb{H}$ we denote by $\arg (u)$ the unique number satisfying $\arg (u) \in(0,1], u=\exp (\mathrm{i} \pi \arg (u))$. It is convenient to set $\arg (0)=-\infty$.
} 
b) Define $K_{0}(\mathcal{T}) \stackrel{Z_{e}}{\longrightarrow} \mathbb{C}$, such that: $K_{0}(\mathcal{A}) \stackrel{K_{0}(\mathcal{A C T})}{\longrightarrow} K_{0}(\mathcal{T}) \stackrel{Z_{e}}{\longrightarrow} \mathbb{C}=K_{0}(\mathcal{A}) \stackrel{Z}{\longrightarrow} \mathbb{C}$ is a stability condition on $\mathcal{T}$ (furthermore all stability conditions on $\mathcal{T}$ are obtained by this procedure). Let us denote:

Definition 3.2. Let $\mathcal{A} \subset \mathcal{T}$ be a bounded t-structure in a triangulated category $\mathcal{T}$. We denote by $\mathbb{H}^{\mathcal{A}}$ the family of stability conditions in $\mathcal{T}$ obtained by $\boldsymbol{a})$, $\boldsymbol{b}$ ) above varying $Z$ in the set of all stability functions on $\mathcal{A}$ with $H N$ property. In particular $\mathbb{H}^{\mathcal{A}} \ni(\mathcal{P}, Z) \mapsto Z_{\mid K_{0}(\mathcal{A})}$ is a bijection between $\mathbb{H}^{\mathcal{A}}$ and this set.

Bridgeland proved that the set of all stability conditions, satisfying a property called locally finiteness, on a triangulated category $\mathcal{T}$ is a complex manifold, denoted by $\operatorname{Stab}(\mathcal{T})$.

Remark 3.3. Let $\mathcal{A} \subset \mathcal{T}$ be as in the previous definition. If $\mathcal{A}$ is an abelian category of finite length, then any stability function $Z: K_{0}(\mathcal{A}) \rightarrow \mathbb{C}$ satisfies the $H N$ property ([13, Proposition 2.4]). If in addition $\mathcal{A}$ has finitely many, say $s_{1}, s_{2}, \ldots, s_{n}$, simple objects then all stability conditions in $\mathbb{H}^{\mathcal{A}}$ are locally finite. Whence, in this setting we have $\mathbb{H}^{\mathcal{A}} \subset \operatorname{Stab}(\mathcal{T})$ and bijection $\mathbb{H}^{\mathcal{A}} \ni(\mathcal{P}, Z) \mapsto$ $\left(Z\left(s_{1}\right), \ldots, Z\left(s_{n}\right)\right) \in \mathbb{H}^{n}$.

Finally, we introduce the notation:

Definition 3.4. Let $\mathcal{T}$ be a triangulated category and $\sigma=(\mathcal{P}, Z) \in \operatorname{Stab}(\mathcal{T})$ a stability condition on it. We denote 4

$$
P_{\sigma}^{\mathcal{T}}=\exp (\mathrm{i} \pi\{t \in \mathbb{R} \mid \mathcal{P}(t) \neq\{0\}\}) \subset S^{1} .
$$

By $\mathcal{P}(t+1)=\mathcal{P}(t)[1]^{5}$ it follows $-P_{\sigma}^{\mathcal{T}}=P_{\sigma}^{\mathcal{T}}$.

3.1.2. On $\theta$-stability and a theorem by King. In the next subsection we use a result by King. We recall first

Definition $3.5\left(\theta\right.$-stability). Let $\theta: K_{0}(\mathcal{A}) \rightarrow \mathbb{R}$ be a non-trivial group homomorphism, where $\mathcal{A}$ is an abelian category. Then $X \in \mathcal{A}$ is called $\theta$-semistable if $\theta(X)=0$ and for each monic arrow $X^{\prime} \rightarrow X$ in $\mathcal{A}$ we have $\theta\left(X^{\prime}\right) \geq 0$ (if $\theta\left(X^{\prime}\right)=0$ only for the sub-objects 0 and $X$ then it is called $\theta$-stable).

Remark 3.6. Z-semistable of phase $t$ (as defined in Definition 3.1) is the same as $\theta$-semistable with $\theta=-\operatorname{Im}\left(\mathrm{e}^{-\mathrm{i} \pi t} Z\right)$.

From Proposition 4.4 in [33] it follows

Proposition 3.7 (A. King). Let $A$ be a finite dimensional, hereditary $\mathbb{C}$-algebra. Let $\alpha \in K_{0}(A$-Mod). Then the following conditions are equivalent:

(1) There exist $X \in A$-Mod and a non-trivial $\theta: K_{0}(A$-Mod $) \rightarrow \mathbb{R}$, s. $t .[X]=\alpha$ and $X$ is $\theta$-stable.

(2) $\alpha$ is a Schur root, which by definition means that some $Y \in A$-Mod with $[Y]=\alpha$ satisfies $\operatorname{End}_{A-\operatorname{Mod}}(Y)=\mathbb{C}$.

This Proposition will be used in the proof of Corollary 3.19.

\footnotetext{
${ }^{4}$ When the triangulated category $\mathcal{T}$ is fixed in advance we write just $P_{\sigma}$.

5 which is one of the Bridgeland's axioms
} 
3.2. Dynkin, Euclidean quivers and Kronecker quiver. In this subsection we comment the set $P_{\sigma}$ as $\sigma$ varies in the set of stability conditions on Dynkin, Euclidean quivers and on the Kronecker quiver. The main results here are Lemma 3.13, Corollary 3.15 and Corollary 3.20.

3.2.1. Quivers and Kac's theorem. For any quiver $Q$ we denote its set of vertices by $V(Q)$, its set of arrows by $\operatorname{Arr}(Q)$ and the underlying non-oriented graph by $\Gamma(Q)$. Let

$$
\operatorname{Arr}(Q) \rightarrow V(Q) \times V(Q) \quad a \mapsto(s(a), t(a)) \in V(Q) \times V(Q)
$$

be the function assigning to an arrow $a \in \operatorname{Arr}(Q)$ its origin $s(a) \in V(Q)$ and its end $t(a) \in V(Q)$. A vertex $v \in V(Q)$ is called source/sink if all arrows touching it start/end at it (more precisely $v \neq t(a) / v \neq s(a)$ for each $a \in \operatorname{Arr}(Q))$.

Throughout this section 3 the term Dynkin quiver means a quiver $Q$, s. t. $\Gamma(Q)$ is one of the simply laced Dynkin diagrams $A_{m}, m \geq 1, D_{m}, m \geq 4, E_{6}, E_{7}, E_{8}$ (see for example [5, p. 32]) and the term Euclidean quiver means an acyclic quiver $Q$, s. t. $\Gamma(Q)$ is one of the extended Dynkin diagrams $\widetilde{A}_{m}, m \geq 1, \widetilde{D}_{m}, m \geq 4, \widetilde{E}_{6}, \widetilde{E}_{7}, \widetilde{E}_{8}$ (see for example [5, fig. (4.13)]). By $K(l), l \geq 1$ we denote the quiver, which consists of two vertices with $l$ parallel arrows between them. Note that $K(1)$ is Dynkin, $K(2)$ is Euclidean. We call $K(l), l \geq 3$ and $l$-Kronecker quiver.

Recall the Kac's Theorem.

Remark 3.8 (On Kac's Theorem). Let $Q$ be a connected quiver without edges-loops. In [30] is defined the positive root system of $Q$. We denote this root system by $\Delta_{+}(Q) \subset \mathbb{N}^{V(Q)}$. For $X \in \operatorname{Rep}_{\mathbb{C}}(Q)$ we denote by $\underline{\operatorname{dim}}(X) \in \mathbb{N}^{V(Q)}$ its dimension vector. The main result of [30] (we consider only the field $\mathbb{C})$ is:

$$
\left\{\underline{\operatorname{dim}}(X) \mid X \in \operatorname{Rep}_{\mathbb{C}}(Q), X \text { is indecomposable }\right\}=\Delta_{+}(Q) .
$$

The Euler form of any quiver $Q$ is defined by

$$
\langle\alpha, \beta\rangle_{Q}=\sum_{j \in V(Q)} \alpha_{j} \beta_{j}-\sum_{j \in Q_{1}} \alpha_{s(j)} \beta_{t(j)}, \quad \alpha, \beta \in \mathbb{N}^{V(Q)} .
$$

The set $\Delta_{+}(Q)$ has a simple description for Dynkin, extended Dynkin or hyperbolic quivers $(K(l), l \geq 3$ are hyperbolic quivers) as shown by Kac in [30]. It is determined by the Euler form as follows

$$
\Delta(Q)=\left\{r \in \mathbb{N}^{V(Q)} \backslash\{0\} \mid\langle r, r\rangle_{Q} \leq 1\right\}, \quad \Delta_{+}(Q)=\Delta(Q) \cap \mathbb{N}^{V(Q)} .
$$

If $Q$ has no oriented cycles, (then $Q$ is called acyclic and the path algebra $\mathbb{C} Q$ is finite dimensional) in addition to this we have an isomorphism $K_{0}\left(\operatorname{Rep}_{\mathbb{C}}(Q)\right) \cong \mathbb{Z}^{V(Q)}$ determined by $K_{0}\left(\operatorname{Rep}_{\mathbb{C}}(Q)\right) \ni[X] \mapsto \underline{\operatorname{dim}}(X) \in \mathbb{Z}^{V(Q)}$, where $X \in \operatorname{Rep}_{\mathbb{C}}(Q)$. In particular, for any homomorphism $Z: K_{0}\left(\operatorname{Rep}_{\mathbb{C}}(Q)\right) \rightarrow \mathbb{C}$ and any $X \in \operatorname{Rep}_{\mathbb{C}}(Q)$ we have

$$
Z(X)=\sum_{i \in V(Q)} \underline{\operatorname{dim}}_{i}(X) Z\left(s_{i}\right)=(v, \underline{\operatorname{dim}}(X)), \quad\left\{v_{i}=Z\left(s_{i}\right)\right\}_{i \in V(Q)},
$$

where $s_{i}$ is the simple representation with $\mathbb{C}$ in the vertex $i \in V(Q)$ and 0 in the other vertices. Throughout this section $3($,$) denotes the bilinear form on \mathbb{C}^{V(Q)} \times \mathbb{C}^{V(Q)}$ defined by $(\alpha, \beta)=$ $\sum_{i \in V(Q)} \alpha_{i} \beta_{i}, \alpha, \beta \in \mathbb{C}^{V(Q)}$, NOT the symmetrization $\langle\alpha, \beta\rangle_{Q}+\langle\beta, \alpha\rangle_{Q}$ of $\langle,\rangle_{Q}$. We mention once this symmetrization and denote it by $(,)_{Q}$. 
3.2.2. The inclusion $P_{\sigma} \subseteq R_{v, \Delta_{+}}$.

Lemma 3.9. Let $\mathcal{T}$ be any triangulated category. Then for each $\sigma=(\mathcal{P}, Z) \in \operatorname{Stab}(\mathcal{T})$ we have: $\{t \in \mathbb{R} \mid \mathcal{P}(t) \neq 0\}=\left\{\phi_{\sigma}(I) \mid I\right.$ is $\mathcal{T}$-indecomposable and $\sigma$-semistable $\}$.

Proof. Let $X \in \mathcal{P}(t)$ be non-zero. Since $\mathcal{P}(t)$ is of finite length then we have a decomposition in $\mathcal{P}(t)$ of the form $X \cong \bigoplus_{i=1}^{n} X_{i}$, where $X_{i}$ are indecomposable in $\mathcal{P}(t)$, therefore (here we use that $\mathcal{P}(t)$ is abelian) there are not non-trivial idempotents in $\operatorname{End}_{\mathcal{P}(t)}\left(X_{i}\right)=\operatorname{End}_{\mathcal{T}}\left(X_{i}\right)$, hence $X_{i}$ is indecomposable in $\mathcal{T}$. Whence, we see that $t=\phi_{\sigma}\left(X_{i}\right)$, where $X_{i}$ is an indecomposable in $\mathcal{T}$ and $\sigma$-semistable. The lemma follows.

Corollary 3.10. Let $\mathcal{A}$ be a hereditary abelian category. For each $\sigma=(\mathcal{P}, Z) \in \operatorname{Stab}\left(D^{b}(\mathcal{A})\right)$ holds the inclusion:

$$
P_{\sigma} \subseteq\left\{ \pm \frac{Z(X)}{|Z(X)|} \mid X \text { is indecomposable in } \mathcal{A}, Z(X) \neq 0\right\}
$$

Proof. Take any $t \in \mathbb{R}$ with $\mathcal{P}(t) \neq\{0\}$. From the previous lemma there is a semi-stable, indecomposable $X \in D^{b}(\mathcal{A})$, s. t. $\phi_{\sigma}(X)=t$. Since $\mathcal{A}$ is hereditary then $X=X^{\prime}[i]$ for some indecomposable $X^{\prime} \in \mathcal{A}, i \in \mathbb{Z}$. Now we can write

$$
(-1)^{i} Z\left(X^{\prime}\right)=Z(X)=m(X) \exp \left(\mathrm{i} \pi \phi_{\sigma}(X)\right)=m(X) \exp (\mathrm{i} \pi t) \quad m(X)>0,
$$

where we use that $X$ is $\sigma$-semistable and one of the Bridgeland's axioms ([13, Definition 1.1 a)]). The corollary is proved.

When $\mathcal{A}=\operatorname{Rep}_{\mathbb{C}}(Q)$ with $Q$-acyclic we can rewrite this corollary in a useful form. Putting (62) and $(65)$ in the righthand side of $(66)$ with $\mathcal{A}=\operatorname{Rep}_{\mathbb{C}}(Q)$ we get a set $\left\{ \pm \frac{(v, r)}{|(v, r)|} \mid r \in \Delta_{+}(Q),(v, r) \neq 0\right\}$, where $v \in \mathbb{C}^{V(Q)}$ is a non-zero vector. It is useful to define

Definition 3.11. For any finite set $F$, any subset $A \subset \mathbb{N}^{F} \backslash\{0\}$ and any non-zero vector $v \in \mathbb{C}^{F}$ we denote 6

$$
R_{v, A}^{F}=\left\{ \pm \frac{(v, r)}{|(v, r)|} \mid r \in A,(v, r) \neq 0\right\} \subset S^{1}, \text { where }(v, r)=\sum_{i \in F} v_{i} r_{i} .
$$

Then we can rewrite (66) as follows (we assume that $Q$ is an acyclic, because we used (65), which holds only for acyclic quivers) :

Corollary 3.12. Let $Q$ be an acyclic quiver. For any $\sigma=(\mathcal{P}, Z) \in \operatorname{Stab}\left(D^{b}\left(\operatorname{Rep}_{\mathbb{C}}(Q)\right)\right)$ holds the inclusion

$$
P_{\sigma} \subseteq R_{v, \Delta_{+}(Q)} \quad v=\left\{v_{i}=Z\left(s_{i}\right)\right\}_{i \in V(Q)} .
$$

3.2.3. On the set $R_{v, \Delta_{+}(Q)}$.

Lemma 3.13. Let $Q$ be a Dynkin quiver. For any stability condition $\sigma \in \operatorname{Stab}\left(D^{b}\left(\operatorname{Re}_{\mathbb{C}}(Q)\right)\right)$ the set of semi-stable phases $P_{\sigma}$ is finite.

Proof. It is well known that for a Dynkin quiver $Q$ the positive root system $\Delta_{+}(Q)$ is finite. Hence for any non-zero $v \in \mathbb{C}^{V(Q)}$ the set $R_{v, \Delta_{+}(Q)}$ is finite. Now the lemma follows from Corollary 3.12

\footnotetext{
${ }^{6}$ When the set $F$ is clear we write just $R_{v, A}$.
} 
Lemma 3.14. Let $Q$ be an Euclidean quiver (see subsubsection 3.2.1 for definition). For any non-zero $v \in \mathbb{C}^{V(Q)}$ the set $R_{v, \Delta_{+}(Q)}$ is either finite or there exist $m \in \mathbb{N}, p \in S^{1}$ and sequences $\left\{p_{j}^{i} \subset S^{1}\right\}_{i=1, \ldots, m ; j \in \mathbb{N}}$, s. t. $\left\{\lim _{j \rightarrow \infty} p_{j}^{i}=p\right\}_{i=1}^{m}$ and $R_{v, \Delta_{+}}=\cup_{i=1}^{m}\left\{ \pm p_{j}^{i}\right\}_{j \in \mathbb{N}}$.

Proof. The root system $\Delta$ of an Euclidean quiver $Q$ (as described in the first equality of (64) has an element $\delta \in \mathbb{N}_{\geq 1}^{V(Q)}$ with the properties $\Delta \cup\{0\}+\mathbb{Z} \delta \subset \Delta \cup\{0\}$ and $\Delta \cup\{0\} / \mathbb{Z} \delta$ is finite (see [18, p. 18]). Hence there is a finite set $\left\{\alpha_{1}, \alpha_{2} \ldots, \alpha_{m}\right\} \subset \Delta$, s. t. $\Delta \cup\{0\}=\bigcup_{i=1}^{m}\left(\alpha_{i}+\mathbb{Z} \delta\right)$. If for any $i \in\{1,2, \ldots, m\}$ we choose the minimal $n_{i} \in \mathbb{Z}$, s. t. $\alpha_{i}+n_{i} \delta \in \Delta_{+}$and denote $\beta_{i}=\alpha_{i}+n_{i} \delta$, then $\Delta_{+}=\bigcup_{i=1}^{m}\left(\beta_{i}+\mathbb{N} \delta\right)$. From the definition 67) of $R_{v, \Delta_{+}}$we see that

$$
R_{v, \Delta_{+}}=\bigcup_{i=1}^{m}\left\{ \pm \frac{\left(v, \beta_{i}\right)+n(v, \delta)}{\left|\left(v, \beta_{i}\right)+n(v, \delta)\right|} \mid n \in \mathbb{N}, i=1,2, \ldots, m,\left(v, \beta_{i}\right)+n(v, \delta) \neq 0\right\} .
$$

If $(v, \delta)=0$, then the set is finite. Otherwise for $i=\{1,2, \ldots, m\}$ we have $\lim _{n \rightarrow \infty} \frac{\left(v, \beta_{i}\right)+n(v, \delta)}{\left|\left(v, \beta_{i}\right)+n(v, \delta)\right|}=$ $\frac{(v, \delta)}{|(v, \delta)|}$.

From this lemma and Corollary 3.12 it follows:

Corollary 3.15. Let $Q$ be an Euclidean quiver. Then for any $\sigma \in D^{b}\left(\operatorname{Rep}_{k}(Q)\right)$ the set $P_{\sigma}$ is either finite or has exactly two limit points of the type $\{p,-p\} 7$.

Proof. If $P_{\sigma}$ is infinite, then by the previous lemma and $P_{\sigma} \subset R_{v, \Delta_{+}},\left\{v_{i}=Z\left(s_{i}\right)\right\}_{i \in V(Q)}$ it follows that $R_{v, \Delta_{+}}=\cup_{i=1}^{m}\left\{ \pm p_{j}^{i}\right\}_{j \in \mathbb{N}}$ with $\lim _{j \rightarrow \infty} p_{j}^{i}=p$ for $i=1,2, \ldots, m$. In particular $P_{\sigma}$ can not have more than two limit points. Since $P_{\sigma}$ is infinite then $P_{\sigma} \cap\left\{p_{j}^{i}\right\}_{j \in \mathbb{N}}, P_{\sigma} \cap\left\{-p_{j}^{i}\right\}_{j \in \mathbb{N}}$ are infinite sets for some $i$ (recall that $-P_{\sigma}=P_{\sigma}$ ). Hence $\{p,-p\}$ are limit points of $P_{\sigma}$. The corollary follows.

Next we discuss the set $R_{v, \Delta_{+}}$for the $l$-Kronecker quiver $K(l)$ (two vertices with $l$ parallel arrows between them), $l \geq 3$. In this case the vertices are two, so that $v$ has two complex coordinates. I.e. $R_{v, \Delta_{+}}$consists of fractions like $\frac{n z_{1}+m z_{2}}{\left|n z_{1}+m z_{2}\right|}$, where $z_{1}, z_{2} \in \mathbb{C}, n, m \in \mathbb{N}$. It is useful to note

Remark 3.16. Let $z_{i}=r_{i} \exp \left(\mathrm{i} \phi_{i}\right), r_{i}>0, i=1,2,0<\phi_{2}<\phi_{1} \leq \pi$. Then

$$
\frac{\alpha z_{1}+\beta z_{2}}{\left|\alpha z_{1}+\beta z_{2}\right|}=\left\{\begin{array}{cc}
\exp \left(\mathrm{i} f\left(\frac{\alpha}{\beta}\right)\right) & \alpha \geq 0, \beta>0, \\
\exp \left(\mathrm{i} \phi_{1}\right) & \alpha>0, \beta=0,
\end{array}\right.
$$

where $f:[0, \infty) \rightarrow\left[\phi_{2}, \phi_{1}\right) \subset(0, \pi)$ is the strictly increasing smooth function:

$$
f(x)=\arccos \left(\frac{x r_{1} \cos \left(\phi_{1}\right)+r_{2} \cos \left(\phi_{2}\right)}{\sqrt{x^{2} r_{1}^{2}+r_{2}^{2}+2 x r_{1} r_{2} \cos \left(\phi_{1}-\phi_{2}\right)}}\right), \quad f(0)=\phi_{2}, \lim _{x \rightarrow \infty} f(x)=\phi_{1} .
$$

From (63) we see that the Euler form for the quiver $K(l)$ is $\left\langle\left(\alpha_{1}, \alpha_{2}\right),\left(\beta_{1}, \beta_{2}\right)\right\rangle_{K(l)}=\alpha_{1} \beta_{1}+$ $\alpha_{2} \beta_{2}-l \alpha_{1} \beta_{2}$. Hence the positive roots are

$$
\Delta_{l+}=\Delta_{+}(K(l))=\left\{(n, m) \in \mathbb{N}^{2} \mid n^{2}+m^{2}-\operatorname{lm} n \leq 1\right\} \backslash\{(0,0)\} .
$$

Remark 3.17. Since the root systems $\Delta(K(l))$ with $l \geq 3$ will play an important role we reserve for them the notation $\Delta_{l}=\Delta(K(l))$, respectively $\Delta_{l+}=\Delta_{+}(K(l))$.

\footnotetext{
${ }^{7} \operatorname{In}\left[20\right.$ are shown examples of both the cases: $P_{\sigma}$ is finite and $P_{\sigma}$ is with two limit points.
} 
The roots with $n^{2}+m^{2}-l m n=1$ are called real roots and with $n^{2}+m^{2}-l m n \leq 0$ - imaginary roots. We can represent the real and the imaginary roots as follows:

$$
\begin{gathered}
\Delta_{l+}^{r e}=\{(1,0)\} \cup\{(0,1)\} \cup\left\{\frac{n}{m}=\frac{1}{2}\left(l \pm \sqrt{l^{2}-4+\frac{4}{m^{2}}}\right) \mid n, m \in \mathbb{N}_{\geq 1},(n, m)=1\right\} \\
\Delta_{l+}^{i m}=\left\{\frac{1}{2}\left(l-\sqrt{l^{2}-4}\right) \leq \frac{n}{m} \leq \frac{1}{2}\left(l-\sqrt{l^{2}-4}\right) \mid n \in \mathbb{N}_{\geq 0}, m \in \mathbb{N}_{\geq 1}\right\} .
\end{gathered}
$$

Lemma 3.18. Let $v=\left(z_{1}, z_{2}\right), z_{i}=r_{i} \exp \left(\mathrm{i} \phi_{i}\right), r_{i}>0,0<\phi_{2}<\phi_{1} \leq \pi$. Let us denote $u=f\left(\frac{1}{2}\left(l-\sqrt{l^{2}-4}\right)\right), v=f\left(\frac{1}{2}\left(l+\sqrt{l^{2}-4}\right)\right)$, where $f$ is defined in Remark 3.16. Then

$$
R_{v, \Delta_{l+}}=\left\{ \pm c_{j}\right\}_{j \in \mathbb{N}} \cup \pm D \cup\left\{ \pm a_{j}\right\}_{j \in \mathbb{N}}
$$

where $D$ is a dense subset in the arc $\exp (\mathrm{i}[u, v]) \subset S^{1},\left\{a_{j}\right\}_{j \in \mathbb{N}}$ is a sequence with $a_{0}=\exp \left(\mathrm{i} \phi_{2}\right)$ and anti-clockwise monotonically converges to $\exp (\mathrm{i} u),\left\{c_{j}\right\}_{j \in \mathbb{N}}$ is a sequence with $c_{0}=\exp \left(\mathrm{i} \phi_{1}\right)$ and clockwise monotonically converging to $\exp (\mathrm{i} v)$.

Proof. Now $R_{v, \Delta_{l+}}=\left\{ \pm \frac{n z_{1}+m z_{2}}{\left|n z_{1}+m z_{2}\right|} \mid(n, m) \in \Delta_{l+}\right\}$. We have a disjoint union $\Delta_{l+}=\Delta_{l+}^{r e} \cup \Delta_{l+}^{i m}$, where $\Delta_{l+}^{r e}, \Delta_{l+}^{i m}$ are taken from (73), (74). Recall also that if $m \geq 1$ then $\frac{n z_{1}+m z_{2}}{\left|n z_{1}+m z_{2}\right|}=\exp (\mathrm{i} f(n / m))$ (see Remark 3.16). Therefore we can write for $R_{v, \Delta_{l+}}$ :

$$
\begin{gathered}
\left\{ \pm \frac{n z_{1}+m z_{2}}{\left|n z_{1}+m z_{2}\right|} \mid(n, m) \in \Delta_{l+}^{r e}\right\} \cup\left\{ \pm \frac{n z_{1}+m z_{2}}{\left|n z_{1}+m z_{2}\right|} \mid(n, m) \in \Delta_{l+}^{i m}\right\}= \\
\left\{ \pm \exp \left(\mathrm{i} \phi_{1}\right)\right\} \cup\left\{ \pm \exp \left(\mathrm{i} \phi_{2}\right)\right\} \cup\left\{ \pm \exp \left(\mathrm{i} f\left(\frac{1}{2}\left(l \pm \sqrt{l^{2}-4+\frac{4}{m^{2}}}\right)\right)\right) \mid m \in \mathbb{N}_{\geq 1}\right\} \\
\cup\{ \pm \exp (\mathrm{i} f(n / m)) \mid n / m \in[u, v]\} .
\end{gathered}
$$

Now the lemma follows from the properties of $f$ given in Remark 3.16 and the fact that $\mathbb{Q} \cap[u, v]$ is dense in $[u, v]$.

3.2.4. Stability conditions $\sigma$ on $K(l)$ with $P_{\sigma}=R_{v, \Delta_{l+}}$. In this subsection $l \geq 3$ is fixed and $Q=K(l), \Delta_{l+}$ is the positive root system of $K(l), \mathcal{A}=\operatorname{Rep}_{\mathbb{C}}(Q), \mathcal{T}=D^{b}\left(\operatorname{Rep}_{\mathbb{C}}(Q)\right)$.

For a representation $X=\mathbb{C}^{n} \quad \vdots \quad \mathbb{C}^{m} \in \mathcal{A}$ we write $\underline{\operatorname{dim}}(X)=(n, m), \underline{\operatorname{dim}}_{1}(X)=n$,

$\underline{\operatorname{dim}}_{2}(X)=m$. The simple objects of the standard t-structure $\mathcal{A}=\operatorname{Rep}_{\mathbb{C}}(Q) \subset D^{b}\left(\operatorname{Rep}_{\mathbb{C}}(Q)\right)$ are:

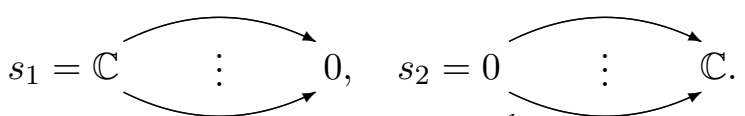

To $\mathcal{A} \subset \mathcal{T}$ we can apply Remark 3.3 and then we have $\mathbb{H}^{\mathcal{A}} \subset \operatorname{Stab}(\mathcal{T})$ and bijection $\mathbb{H}^{\mathcal{A}} \ni(\mathcal{P}, Z) \mapsto$ $\left(Z\left(s_{1}\right), Z\left(s_{2}\right)\right) \in \mathbb{H}^{2}$.

For any $(\mathcal{P}, Z) \in \mathbb{H}^{\mathcal{A}}, t \in(0,1] \mathcal{P}(t)$ consists of the objects in $\mathcal{A}$ satisfying the condition in Definition 3.1. If we denote $v=\left(Z\left(s_{1}\right), Z\left(s_{2}\right)\right) \in \mathbb{H}^{2}$, then by $Z(X)=(v, \underline{\operatorname{dim}}(X))$ :

$$
X \in \mathcal{P}(t), t \in(0,1] \Longleftrightarrow
$$

for any $\mathcal{A}$-monic $\quad X^{\prime} \rightarrow X \quad \arg \left(v, \underline{\operatorname{dim}}\left(X^{\prime}\right)\right) \leq \arg (v, \underline{\operatorname{dim}}(X))=\pi t$. 
Lemma 3.19. Let $\sigma=(\mathcal{P}, Z) \in \mathbb{H}^{\mathcal{A}}$ and $\arg \left(Z\left(s_{1}\right)\right)>\arg \left(Z\left(s_{2}\right)\right)$. Let $(n, m) \in \Delta_{l+}$ be a Schur root. Then $\frac{n z_{1}+m z_{2}}{\left|n z_{1}+m z_{2}\right|} \in P_{\sigma}$, where $z_{i}=Z\left(s_{i}\right), i=1,2$.

Proof. So, let $(n, m) \in \Delta_{l+}$ be a Schur root. We show that there exists a $\sigma$-semistable $X$ with $\underline{\operatorname{dim}}(X)=(n, m)$. Then the lemma follows because $X \in \mathcal{P}(t) \neq\{0\}$ for some $t \in(0,1]$ and by the formula $m(X) \exp (\mathrm{i} \pi t)=Z(X)=n z_{1}+m z_{2}$.

If $m=0$, then $n=1$ (recall $(72)$ ) and then $X=s_{1}$ is the semistable, which we need (it is even stable in $\sigma$, since it is a simple object in $\mathcal{A})$. Hence we can assume that $m \geq 1$.

Denote $\arg \left(z_{i}\right)=\phi_{i}, i=1,2, v=\left(z_{1}, z_{2}\right)$. Then $0<\phi_{2}<\phi_{1} \leq \pi$. By 70 ) for any $X$ with $\underline{\operatorname{dim}}(X)=(n, m)$ we have $\arg (v, \underline{\operatorname{dim}}(X))=\arg \left(n z_{1}+m z_{2}\right)=f(n / m)$. Then by $(76)$ such a $X$ is semi-stable in $\sigma$ iff any $\mathcal{A}$-monic $X^{\prime} \rightarrow X$ satisfies

$$
\arg \left(\underline{\operatorname{dim}}_{1}\left(X^{\prime}\right) z_{1}+\underline{\operatorname{dim}}_{2}\left(X^{\prime}\right) z_{2}\right) \leq f\left(\frac{n}{m}\right) .
$$

Recall that $f(n / m)<\phi_{1}$ (see Remark (3.16)). From the last inequality we get $\underline{\operatorname{dim}}_{2}\left(X^{\prime}\right) \neq 0$ and then by 70 this inequality can be rewritten as $f\left(\underline{\operatorname{dim}}_{1}\left(X^{\prime}\right) / \underline{\operatorname{dim}}_{2}\left(X^{\prime}\right)\right) \leq f(n / m)$.

So, we see that $X \in \mathcal{A}$ with $\underline{\operatorname{dim}}(X)=(n, m)$ is $\sigma$-semistable iff any $\mathcal{A}$-monic arrow $X^{\prime} \rightarrow X$ satisfies:

$$
\underline{\operatorname{dim}}_{2}\left(X^{\prime}\right) \neq 0, \quad \frac{\operatorname{dim}_{1}\left(X^{\prime}\right)}{\underline{\operatorname{dim}}_{2}\left(X^{\prime}\right)} \leq \frac{n}{m} .
$$

Now since $(n, m)$ is a Schur root then by Proposition 3.7 there exists $X \in \mathcal{A}$ with $\underline{\operatorname{dim}}(X)=(n, m)$ and a non-zero $\theta: K_{0}(\mathcal{A}) \rightarrow \mathbb{R}$, s. t. $X$ is $\theta$-semistable (see definition 3.5). We will show that this $X$ is the $\sigma$-semistable, which we need.

By $\theta$-semistability of $X$ we have $\theta(1,0) n+\theta(0,1) m=0$. By $m \neq 0$ we have a monic map ${ }^{8}$ $s_{2} \rightarrow X$ and then again by $\theta$-semistability $\theta(0,1) \geq 0$, which together with $\theta(1,0) n+\theta(0,1) m=0$, $\theta \neq 0$ implies

$$
\theta(1,0)<0, \quad \theta(0,1)>0, \quad \theta(1,0) \frac{n}{m}+\theta(0,1)=0 .
$$

Let us take now any monic arrow $X^{\prime} \rightarrow X$ in $\mathcal{A}$ with $X^{\prime} \neq 0$. By $\theta$-semistability $0 \leq \theta\left(X^{\prime}\right)=$ $\theta(1,0) \underline{\operatorname{dim}}_{1}\left(X^{\prime}\right)+\theta(0,1) \underline{\operatorname{dim}}_{2}\left(X^{\prime}\right)$. Hence by $\theta(1,0)<0$ we obtain $\underline{\operatorname{dim}}_{2}\left(X^{\prime}\right) \neq 0$. Therefore we can write

$$
\theta(1,0) \frac{\operatorname{dim}_{1}\left(X^{\prime}\right)}{\underline{\operatorname{dim}}_{2}\left(X^{\prime}\right)}+\theta(0,1) \geq 0=\theta(1,0) \frac{n}{m}+\theta(0,1) .
$$

By $\theta(1,0)<0$ it follows $\frac{\operatorname{dim}_{1}\left(X^{\prime}\right)}{\underline{\operatorname{dim}}_{2}\left(X^{\prime}\right)} \leq \frac{n}{m}$. Whence, we verified 77 and the lemma follows.

Corollary 3.20. Let $\sigma=(\mathcal{P}, Z) \in \mathbb{H}^{\operatorname{Re} p_{\mathbb{C}}(K(l))} \subset \operatorname{Stab} D^{b}\left(\operatorname{Re} p_{\mathbb{C}}(K(l))\right)$ and $\arg \left(Z\left(s_{1}\right)\right)>\arg \left(Z\left(s_{2}\right)\right)$. Then $P_{\sigma}=R_{v, \Delta_{l+}}$, where $v=\left(Z\left(s_{1}\right), Z\left(s_{2}\right)\right)$.

Proof. The good luck is that all elements of $\Delta_{l+}$ are Schur roots. Indeed [30, Theorem 4 a)] says that $\Delta_{l+}^{i m}$ are Schur roots. In [45] one can read that the indecomposable representations of $Q$ with dimension vectors real roots are also Schur. Whence, we see that any $(n, m) \in \Delta_{l+}$ is a Schur root and we can apply the previous lemma to it. The corollary follows.

Remark 3.21. Recently it was noted in [28] that there is a connection between [50], [51, [43] and the density in an arc for the Kronecker quiver.

\footnotetext{
${ }^{8}$ Since the vertex corresponding to $s_{2}$ is a sink, then $s_{2}$ is a subobject of any $X \in \operatorname{Re} p_{\mathbb{C}}(K(l))$ with $\underline{\operatorname{dim}}_{2}(X) \neq 0$.
} 
3.3. Kronecker pairs. In this subsection we generalize Corollary 3.20, The most general statement is Theorem 3.27, but we use further only its Corollary 3.29 (corollary 3.28 is intermediate). The first step is: 9

Lemma 3.22. Let $\mathcal{T}$ be a $k$-linear triangulated category, where $k$ is any field. Let $\left(E_{1}, E_{2}\right)$ be a full exceptional pair, s. $t$. $\operatorname{Hom}^{\leq 0}\left(E_{1}, E_{2}\right)=0,0<\operatorname{dim}_{k}\left(\operatorname{Hom}^{1}\left(E_{1}, E_{2}\right)\right)=l<\infty$. Let $\mathcal{A}$ be the extension closure of $\left(E_{1}, E_{2}\right)$ in $\mathcal{T}$.

Then $\mathcal{A}$ is a heart of a bounded t-structure in $\mathcal{T}$ and there exists an equivalence of abelian categories: $F: \mathcal{A} \rightarrow \operatorname{Rep}_{k}(K(l))$, s. t. $F\left(E_{1}\right)=s_{1}, F\left(E_{2}\right)=s_{2} \quad\left(s_{1}, s_{2}\right.$ are as in (75)).

Proof. In [17, p. 6] or [44, section 3] it is shown that by hom $\leq 0\left(E_{1}, E_{2}\right)=0$ and 10 T $=\left\langle E_{1}, E_{2}\right\rangle$ it follows that $\mathcal{A}$ is a heart of a bounded t-structure of $\mathcal{T}$ (see also [32, section 8]). In particular $\mathcal{A}$ is an abelian category.

Let $D T(\mathcal{T})$ be the category of distinguished triangles in $\mathcal{T}$ (objects are the distinguished triangles and morphisms are triple of arrows between triangles making commutative the corresponding diagram). Using the semi orthogonal decomposition $\mathcal{T}=\left\langle\left\langle E_{1}\right\rangle,\left\langle E_{2}\right\rangle\right\rangle$ one can construct three functors:

$$
G: \mathcal{T} \rightarrow D T(\mathcal{T}), \quad \lambda_{1}: \mathcal{T} \rightarrow\left\langle E_{1}\right\rangle, \quad \lambda_{2}: \mathcal{T} \rightarrow\left\langle E_{2}\right\rangle
$$

s. t. the triangle $G(X) \in D T(\mathcal{T})$ for any $X \in \mathcal{T}$ is:

$$
G(X)=\lambda_{2}(X) \stackrel{u_{X}}{\longrightarrow} X \stackrel{v_{X}}{\longrightarrow} \lambda_{1}(X) \stackrel{w_{X}}{\longrightarrow} \lambda_{2}(X)[1], \quad \lambda_{1}(X) \in\left\langle E_{1}\right\rangle, \lambda_{2}(X) \in\left\langle E_{2}\right\rangle .
$$

It is well known that $\lambda_{2}$ is right adjoint to the embedding functor $\left\langle E_{2}\right\rangle \rightarrow \mathcal{T}$ and $\lambda_{1}$ is left adjoint to $\left\langle E_{1}\right\rangle \rightarrow \mathcal{T}$ (see for example [29, p. 279]). The adjoint functor (left or right) to an exact functor is also an exact functor (7, Proposition 1.4]). Therefore $\lambda_{1}$ and $\lambda_{2}$ are exact functors. If we restrict $\lambda_{1}, \lambda_{2}$ to $\mathcal{A}$ then we obtain exact functors between abelian categories

$$
\lambda_{i}^{\mathcal{A}}: \mathcal{A} \rightarrow \mathcal{A}_{i} \quad i=1,2,
$$

where $\mathcal{A}_{i} \cong k$-Vect is the additive closure of $E_{i}$.

We define the functor $F: \mathcal{A} \rightarrow \operatorname{Rep}_{k}(K(l))$ as follows. First choose a basis of $\operatorname{Hom}^{1}\left(E_{1}, E_{2}\right)$ and a decomposition of any $Y \in \mathcal{A}_{i}$ into $\operatorname{dim}\left(\operatorname{Hom}\left(E_{i}, Y\right)\right)$ number of copies of $E_{i}, i=1,2$. Take any $X \in \mathcal{A}$, then we get a distinguished triangle $G(X)$ as in $(79)$ with $\lambda_{i}(X)=\lambda_{i}^{\mathcal{A}}(X)$, in particular we get an arrow $\lambda_{1}^{\mathcal{A}}(X) \stackrel{w_{X}}{\longrightarrow} \lambda_{2}^{\mathcal{A}}(X)[1]$. This arrow, using the chosen decompositions and the basis of $\operatorname{Hom}^{1}\left(E_{1}, E_{2}\right)$, can be expressed by $l a_{2} \times a_{1}$ matrices over $k$, where $a_{i}=\operatorname{dim}\left(E_{i}, \lambda_{i}(X)\right), i=1,2$. In particular these $l$ matrices are a representation of $K(l)$ with dimension vector $\left(a_{1}, a_{2}\right)$ and we define $F(X)$ to be this representation.

Let $f: X \rightarrow Y$ be an arrow in $\mathcal{A}$ then, as far as $G: \mathcal{T} \rightarrow D T(\mathcal{T})$ is a functor, $G(f)$ is a morphism of

$$
\lambda_{1}^{\mathcal{A}}(X) \stackrel{w_{X}}{\longrightarrow} \lambda_{2}^{\mathcal{A}}(X)[1]
$$

triangles, hence the diagram: $\lambda_{1}^{\mathcal{A}}(f) \downarrow \quad \lambda_{2}^{\mathcal{A}}(f)[1] \downarrow \quad$ is commutative. Let $M_{1}, M_{2}$ be the matrices

$$
\lambda_{1}^{\mathcal{A}}(Y) \stackrel{w_{Y}}{\longrightarrow} \lambda_{2}^{\mathcal{A}}(Y)[1]
$$

\footnotetext{
${ }^{9}$ It is motivated by the Bondal's result in [9] for equivalence between triangulated category generated by a strong exceptional collection and the derived category of modules over an algebra of homomorphisms of this collection and by a note on this equivalence in 39. Observe however that we do not have restriction on $\left(E_{1}, E_{2}\right)$ to be a strong pair and we construct equivlanece between $t$-structures.

${ }^{10}$ If $S$ is a subset of objects in a triangulated category $\mathcal{T}$ we denote by $\langle S\rangle$ the triangulated subcategory generated by $S$.
} 
of $\lambda_{1}^{\mathcal{A}}(f), \lambda_{2}^{\mathcal{A}}(f)$. The commutativity of the diagram above implies that $\left(M_{1}, M_{2}\right): F(X) \rightarrow F(Y)$ is an arrow in $\operatorname{Rep}_{k}(K(l))$ and our definition of $F(f)$ is $F(f)=\left(M_{1}, M_{2}\right)$.

By the exactness of $\lambda_{i}^{\mathcal{A}}, i=1,2$ it follows that $F$ is an exact functor between abelian categories. Now, by straightforward computations one can show that $F$ is an equivalence.

This lemma prompts the following definition

Definition 3.23. A pair of objects $\left(E_{1}, E_{2}\right)$ in a $k$-linear ( $k$ is any field) triangulated category $\mathcal{T}$ is called Kronecker pair if:

- $\left(E_{1}, E_{2}\right)$ is an exceptional pair

- $\operatorname{Hom} \leq 0\left(E_{1}, E_{2}\right)=0$

- $3 \leq \operatorname{dim}_{k}\left(\operatorname{Hom}^{1}\left(E_{1}, E_{2}\right)\right)<\infty$.

Corollary 3.24. Let $\left(E_{1}, E_{2}\right)$ be a Kronecker pair in a $\mathbb{C}$-linear triangulated category $\mathcal{D}$. Denote $l=\operatorname{dim}\left(\operatorname{Hom}^{1}\left(E_{1}, E_{2}\right)\right), \mathcal{T}=\left\langle E_{1}, E_{2}\right\rangle \subset \mathcal{D}$ and $\mathcal{A}$ - the extension closure of $\left(E_{1}, E_{2}\right)$.

Then any $\sigma=(\mathcal{P}, Z) \in \mathbb{H}^{\mathcal{A}} \subset \operatorname{Stab}^{b}(\mathcal{T})$ with $\arg \left(Z\left(E_{1}\right)\right)>\arg \left(Z\left(E_{2}\right)\right)$ satisfies $P_{\sigma}=R_{v, \Delta_{l+}}$, where $v=\left(Z\left(E_{1}\right), Z\left(E_{2}\right)\right)$. In particular $P_{\sigma}$ is dense in an arc of non-zero length.

Proof. We take the equivalence $F: \mathcal{A} \rightarrow \operatorname{Rep}_{\mathbb{C}}(K(l))$ constructed in Lemma 3.22, $\mathcal{A} \subset \mathcal{T}$, $\operatorname{Rep}_{\mathbb{C}}(K(l)) \subset D^{b}\left(\operatorname{Rep}_{\mathbb{C}}(K(l))\right)$. This equivalence induces a natural bijection $F^{*}: \mathbb{H}^{R e p_{\mathbb{C}}(K(l))} \rightarrow$ $\mathbb{H}^{\mathcal{A}}$. For $\sigma=(\mathcal{P}, Z) \in \mathbb{H}^{\mathcal{A}}, \sigma^{\prime}=\left(\mathcal{P}^{\prime}, Z^{\prime}\right) \in \mathbb{H}^{\operatorname{Re} p_{\mathbb{C}}(K(l))}$, from $F^{*}\left(\sigma^{\prime}\right)=\sigma$ it follows $Z\left(E_{i}\right)=Z^{\prime}\left(s_{i}\right)$ (because $\left.F\left(E_{i}\right)=s_{i}\right)$ and $P_{\sigma}=P_{\sigma^{\prime}}$. Then the corollary follows from Corollary 3.20 .

Whence in this Corollary we obtained $\sigma \in \operatorname{Stab}\left(\left\langle E_{1}, E_{2}\right\rangle\right)$ with $P_{\sigma}$ dense in a nontrivial arc. To obtain $\sigma^{\prime} \in \operatorname{Stab}(\mathcal{D})$ with such a property, we want to extend the given $\sigma \in \operatorname{Stab}\left(\left\langle E_{1}, E_{2}\right\rangle\right)$ to a stability condition on $\mathcal{D} \supset \mathcal{T}$ in the following sense:

Definition 3.25. Let $\mathcal{T} \subset \mathcal{D}$ be a triangulated subcategory in a triangulated category $\mathcal{D}$. We say that $\sigma=(\mathcal{P}, Z) \in \operatorname{Stab}(\mathcal{T})$ can be extended to $\mathcal{D}$ (or extendable to $\mathcal{D}$ ) if there exists $\sigma_{e}=\left(\mathcal{P}_{e}, Z_{e}\right) \in$ $\operatorname{Stab}(\mathcal{D})$, s. t. $\quad Z_{e} \circ K_{0}(\mathcal{T} \subset \mathcal{D})=Z$ and $\left\{\mathcal{P}(t) \subset \mathcal{P}_{e}(t)\right\}_{t \in \mathbb{R}}$. In this case $\sigma_{e}$ is called extension of $\sigma$.

Remark 3.26. From Definition 3.4 it follows that if $\sigma_{e}$ is an extension of $\sigma$, then $P_{\sigma_{e}} \supset P_{\sigma}$.

By Corollary 3.24 it follows:

Theorem 3.27. Let $\left(E_{1}, E_{2}\right)$ be a Kronecker pair in a $\mathbb{C}$-linear triangulated category $\mathcal{D}$. Denote $l=\operatorname{dim}\left(\operatorname{Hom}^{1}\left(E_{1}, E_{2}\right)\right), \mathcal{T}=\left\langle E_{1}, E_{2}\right\rangle \subset \mathcal{D}$ and $\mathcal{A}$ - the extension closure of $\left(E_{1}, E_{2}\right)$.

Then any $\sigma \in \operatorname{Stab} D^{b}(\mathcal{D})$, which is an extension of a stability condition $(\mathcal{P}, Z) \in \mathbb{H}^{\mathcal{A}} \subset$ $\operatorname{Stab} D^{b}(\mathcal{T})$ with $\arg \left(Z\left(E_{1}\right)\right)>\arg \left(Z\left(E_{2}\right)\right)$ satisfies $P_{\sigma} \supset R_{v, \Delta_{l+}}$, where $v=\left(Z\left(E_{1}\right), Z\left(E_{2}\right)\right)$. In particular $P_{\sigma}$ is dense in an arc of non-zero length.

One setting, where we can extend these stability conditions, is as follows.

Assume that $\left(E_{0}, E_{1}, \ldots, E_{n}\right)$ is a full Ext-exceptional collection ${ }^{11}$ in $\mathcal{D}$. Then for any $0 \leq$ $i<j \leq n$ the extension closure $\mathcal{A}_{i j}$ of $E_{i}, E_{i+1}, \ldots, E_{j}$ is a heart of a bounded $t$-structure in $\mathcal{T}_{i j}=\left\langle E_{i}, E_{i+1}, \ldots, E_{j}\right\rangle \subset \mathcal{D}$ (see [39, Lemma 3.14],[17]), hence we have a corresponding family $\mathbb{H}^{\mathcal{A}_{i j}} \subset \operatorname{Stab}\left(\mathcal{T}_{i j}\right)$. In this setting all stability conditions in $\mathbb{H}^{\mathcal{A}_{i j}}$ are extendable to $\mathcal{D}$. The precise

\footnotetext{
${ }^{11}$ The "Ext-" means $\operatorname{Hom}^{\leq 0}\left(E_{i}, E_{j}\right)=0$ for $0 \leq i<j \leq n$.
} 
statement is (see [20] and [39, Proposition 3.17]) that there is a surjective map ${ }^{12} \pi_{i j}: \mathbb{H}^{\mathcal{A}} \rightarrow \mathbb{H}^{\mathcal{A}_{i j}}$, s. t. for any $\sigma \in \mathbb{H}^{\mathcal{A}_{i j}}, \sigma_{e} \in \mathbb{H}^{\mathcal{A}}$ from $\pi_{i j}\left(\sigma_{e}\right)=\sigma$ it follows that $\sigma_{e}$ is an extension of $\sigma$. Having the desired extensions we obtain by Corollary 3.27 .

Corollary 3.28. Let $\left(E_{0}, E_{1}, \ldots, E_{n}\right)$ be a full Ext-exceptional collection in a $\mathbb{C}$-linear triangulated category $\mathcal{D}$. Let $\left(E_{i}, E_{i+1}\right)$ be a Kronecker pair for some $0 \leq i \leq n-1$. Denote $l=$ $\operatorname{dim}\left(\operatorname{Hom}^{1}\left(E_{i}, E_{i+1}\right)\right)$ and the extension closure of $\left(E_{0}, E_{1}, \ldots, E_{n}\right)$ by $\mathcal{A}$.

Then any $\sigma=(\mathcal{P}, Z) \in \mathbb{H}^{\mathcal{A}}$ with $\arg \left(Z\left(E_{i}\right)\right)>\arg \left(Z\left(E_{i+1}\right)\right)$ satisfies $P_{\sigma} \supset R_{v, \Delta_{l+}}$, where $v=\left(Z\left(E_{i}\right), Z\left(E_{i+1}\right)\right)$. In particular $P_{\sigma}$ is dense in an arc of non-zero length.

Corollary 3.29. Let $\left(E_{0}, E_{1}, \ldots, E_{n}\right)$ be any full exceptional collection in a $\mathbb{C}$-linear triangulated category $\mathcal{D}$ of finite typ $\xi^{13}$. Let $\left(E_{i}, E_{j}\right)$ be a Kronecker pair for some $0 \leq i<j \leq n$. Then there exists a family of stability conditions $\sigma$ on $\mathcal{T}$ for which $P_{\sigma}$ is dense in an arc of non-zero length.

Proof. First by mutations of the exceptional collection $\left(E_{0}, E_{1}, \ldots, E_{n}\right)$ we can obtain a full exceptional collection $\left(E_{i}, E_{j}, C_{2}, \ldots, C_{n}\right)$. Then, because $\mathcal{T}$ is of finite type, after shifts of $C_{2}, C_{3}, \ldots, C_{n}$ we can obtain a full exceptional collection $\mathcal{B}=\left\{B_{0}, B_{2}, \ldots, B_{n}\right\}$, which is Ext and $B_{0}=E_{i}$, $B_{1}=E_{j}$. So we get a full Ext-exceptional collection $\mathcal{B}$ for which $\left(B_{0}, B_{1}\right)$ is a Kronecker pair. Now if we denote by $\mathcal{A}$ the extension closure of $\mathcal{B}$ by Corollary 3.28 it follows that any $\sigma=$ $(\mathcal{P}, Z) \in \mathbb{H}^{\mathcal{A}}$ with $\arg \left(Z\left(B_{0}\right)\right)>\arg \left(Z\left(B_{1}\right)\right)$ satisfies $P_{\sigma} \supset R_{v, \Delta_{l+}}$, where $v=\left(Z\left(B_{0}\right), Z\left(B_{1}\right)\right)$ and $l=\operatorname{dim}_{\mathbb{C}}\left(\operatorname{Hom}^{1}\left(B_{0}, B_{1}\right)\right)$.

Remark 3.30. More general setting, where the stability conditions $\mathbb{H}^{\mathcal{A}}$ in Theorem 3.27 can be extended, is that there exists a semi-orthogonal decomposition $\left(\mathcal{D}^{\prime},\left\langle E_{1}, E_{2}\right\rangle\right)$ of $\mathcal{D}$ with additional assumptions, specified in [17, Theorem 3.6] and [17, Proposition 3.5].

3.4. Application to quivers. In this subsection we apply the results of the previous subsection 3.3 to quivers and obtain Corollary 3.31, Proposition 3.32. Table (1) contains Proposition 3.32 and the results of subsection 3.2 ,

Let $Q$ be an acyclic quiver. The notations $V(Q), \operatorname{Arr}(Q), \Gamma(Q)$ are explained in subsubsection 3.2.1. It is shown in [19] that any exceptional collection $\left(E_{1}, E_{2}, \ldots, E_{n}\right)$ in $\operatorname{Rep}_{\mathbb{C}}(Q)$ of length $n=$ $\#(V(Q))$ is a full exceptional collection of $D^{b}\left(\operatorname{Rep}_{\mathbb{C}}(Q)\right)$. Furthermore, any exceptional collection $\left(E_{1}, E_{2}, \ldots, E_{i}\right)$ in $\operatorname{Rep}_{\mathbb{C}}(Q)$ with $i<n$ can be completed to a full $\left(E_{1}, E_{2}, \ldots, E_{i}, E_{i+1}, \ldots, E_{n}\right)$ exceptional collection. In particular if we are given a Kronecker pair in $\operatorname{Rep}_{\mathbb{C}}(Q)$ we can complete it to a full exceptional collection and, since $D^{b}\left(\operatorname{Rep}_{\mathbb{C}}(Q)\right)$ is of finite type, we can apply Corollary 3.29 . So that only existence of a Kronecker pair in $R e p_{\mathbb{C}}(Q)$ is enough to apply Corollary 3.29 and to construct $\sigma$ with $P_{\sigma}$ dense in an arc. Now using Corollaries 3.13, 3.15, we can easily prove:

Corollary 3.31. Let $Q$ be either an Euclidean or a Dynkin quiver. Then any exceptional pair $\left(E_{1}, E_{2}\right)$ in $\operatorname{Rep}_{\mathbb{C}}(Q)$ satisfies $\operatorname{dim}_{\mathbb{C}}\left(\operatorname{Hom}\left(E_{1}, E_{2}\right)\right)<3, \operatorname{dim}_{\mathbb{C}}\left(\operatorname{Ext}^{1}\left(E_{1}, E_{2}\right)\right)<3$.

Proof. Since $\operatorname{Rep}_{\mathbb{C}}(Q)$ is hereditary then the exceptional objects in $D^{b}\left(\operatorname{Rep}_{\mathbb{C}}(Q)\right)$ are just shifts of exceptional objects in $\operatorname{Rep}_{\mathbb{C}}(Q)$ and then from the arguments above and Corollaries 3.13 , 3.15 it follows that there does not exists a Kronecker pair in $D^{b}\left(\operatorname{Rep}_{\mathbb{C}}(Q)\right)$. In other words for any exceptional pair $\left(E_{1}, E_{2}\right)$ in $D^{b}\left(\operatorname{Rep}_{\mathbb{C}}(Q)\right)$ the minimal nonzero degree $\operatorname{Hom}^{\min }\left(E_{1}, E_{2}\right) \neq 0$,

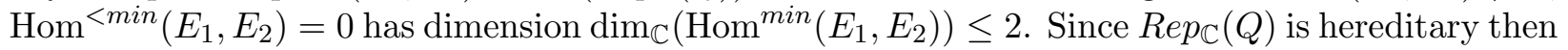

\footnotetext{
${ }^{12}$ We denote here $\mathcal{A}=\mathcal{A}_{0 n}$.

13 by finite type we mean that for any pair $X, Y \in \mathcal{T}$ we have $\sum_{k \in \mathbb{Z}} \operatorname{dim}(\operatorname{Hom}(X, Y[k]))<\infty$.
} 
there are at most two nonzero degrees in $\operatorname{Hom}^{*}\left(E_{1}, E_{2}\right)$ and it remains to show that the maximal nonzero degree $\operatorname{Hom}^{\max }\left(E_{1}, E_{2}\right) \neq 0, \operatorname{Hom}^{>\max }\left(E_{1}, E_{2}\right)=0$ has dimension $\operatorname{dim}_{\mathbb{C}}\left(\operatorname{Hom}^{\max }\left(E_{1}, E_{2}\right)\right) \leq$ 2.

For any exceptional pair $\left(E_{1}, E_{2}\right)$ it is well known that $\left(L_{E_{1}}\left(E_{2}\right), E_{1}\right)$ is also an exceptional pair, where $L_{E_{1}}\left(E_{2}\right)$ is determined by the distinguished triangle:

$$
L_{E_{1}}\left(E_{2}\right) \longrightarrow \operatorname{Hom}^{*}\left(E_{1}, E_{2}\right) \otimes E_{1} \stackrel{e v_{E_{1}, E_{2}}}{\longrightarrow} E_{2} \longrightarrow L_{E_{1}}\left(E_{2}\right)[1] .
$$

Take any $i \in \mathbb{Z}$. We show below that $\operatorname{Hom}^{i}\left(L_{E_{1}}\left(E_{2}\right), E_{1}\right) \cong \operatorname{Hom}^{-i}\left(E_{1}, E_{2}\right)$, which means that the maximal non-zero degree of $\operatorname{Hom}^{*}\left(E_{1}, E_{2}\right)$ is the minimal non-zero degree of $\operatorname{Hom}^{*}\left(L_{E_{1}}\left(E_{2}\right), E_{1}\right)$ and they are isomorphic. Then the corollary follows by the proved inequality for the minimal non-vanishing degrees.

We apply $\operatorname{Hom}^{i}\left({ }_{-}, E_{1}\right)$ to the triangle above and by $\operatorname{Hom}^{*}\left(E_{2}, E_{1}\right)=0$ it follows

$$
\operatorname{Hom}^{i}\left(\operatorname{Hom}^{*}\left(E_{1}, E_{2}\right) \otimes E_{1}, E_{1}\right) \cong \operatorname{Hom}^{i}\left(L_{E_{1}}\left(E_{2}\right), E_{1}\right) .
$$

On the other hand (recall that $E_{1}$ is an exceptional object)

$$
\operatorname{Hom}^{*}\left(E_{1}, E_{2}\right) \otimes E_{1} \cong \bigoplus_{j} E_{1}[-j]^{\operatorname{dim}\left(\operatorname{Hom}^{j}\left(E_{1}, E_{2}\right)\right)} \quad \Rightarrow
$$

$\operatorname{Hom}^{i}\left(\operatorname{Hom}^{*}\left(E_{1}, E_{2}\right) \otimes E_{1}, E_{1}\right) \cong \operatorname{Hom}\left(\oplus_{j} E_{1}[-j]^{\operatorname{dim}\left(\operatorname{Hom}^{j}\left(E_{1}, E_{2}\right)\right)}, E_{1}[i]\right) \cong \mathbb{C}^{\operatorname{dim}\left(\operatorname{Hom}^{-i}\left(E_{1}, E_{2}\right)\right)}$,

which together with 81 give $\operatorname{Hom}^{i}\left(L_{E_{1}}\left(E_{2}\right), E_{1}\right) \cong \operatorname{Hom}^{-i}\left(E_{1}, E_{2}\right)$ and the corollary is proved.

Next we want to prove:

Proposition 3.32. Any acyclic connected quiver $Q$, which is neither Euclidean nor Dynkin has a family of stability conditions $\sigma$ on $D^{b}\left(\operatorname{Rep}_{\mathbb{C}}(Q)\right)$, s. t. $P_{\sigma}$ is dense in an arc of non-zero length.

Remark 3.33. If there are oriented cycles in $Q$, then one can show that there is a famil $\}^{14}\left\{s_{\lambda}\right\}_{\lambda \in \mathbb{C}}$ of non isomorphic simple objects in $\mathcal{A}=\operatorname{Rep}_{\mathbb{C}}(Q)$. Then if we define for simple object $s \in \mathcal{A}$

$$
Z(s)=\left\{\begin{array}{ccc}
\frac{\lambda}{|\lambda|} & \text { if } s=s_{\lambda}, \lambda \in \mathbb{H} \\
\mathrm{i} & \text { otherwise }
\end{array}\right.
$$

we obtain a stability function $Z: K_{0}(\mathcal{A}) \rightarrow \mathbb{C}$, which has $H N$ property, since $\mathcal{A}$ is of finite length. One can show that the corresponding stability condition $\sigma=(\mathcal{P}, Z) \in \mathbb{H}^{\mathcal{A}}$ is locally finite. Since all $\left\{s_{\lambda}\right\}_{\lambda \in \mathbb{C}}$ are simple in $\mathcal{A}$, then they are $\sigma$-semistable. Hence $P_{\sigma}=S^{1}$.

So let us fix a quiver $Q$, satisfying the conditions of Proposition 3.32. By the arguments given in the beginning of this subsection (before Corollary 3.31) we reduce the proof to finding a Kronecker pair in $D^{b}\left(\operatorname{Rep}_{\mathbb{C}}(Q)\right)$. From here till the end of this subsubsection we present the proof of the following:

Proposition 3.34. Any $Q$, satisfying the conditions in Proposition 3.32, has a Kronecker pair in $\operatorname{Rep}_{\mathbb{C}}(Q)$ (i. e. a pair or representations $\left(\rho, \rho^{\prime}\right)$ in $\operatorname{Rep}_{\mathbb{C}}(Q)$ with $\operatorname{Hom}_{\mathcal{T}}^{*}\left(\rho^{\prime}, \rho\right)=\operatorname{Hom}_{\mathcal{T}}^{\leq 0}\left(\rho, \rho^{\prime}\right)=0$, $\operatorname{dim}_{\mathbb{C}}\left(\operatorname{Hom}_{\mathcal{T}}^{1}\left(\rho, \rho^{\prime}\right)\right) \geq 3$, where $\left.\mathcal{T}=D^{b}\left(\operatorname{Rep}_{\mathbb{C}}(Q)\right)\right)$.

\footnotetext{
${ }^{14}$ For example the representations $\{\mathbb{C} \stackrel{\lambda}{\rightarrow} \mathbb{C}\}_{\lambda \in \mathbb{C}}$ of the quiver with one vertex and one loop are all simple and mutually non isomorphic
} 
Recall (see page 8 in [18]) that for $\rho, \rho^{\prime} \in \operatorname{Rep}_{\mathbb{C}}(Q)$, we have the formula

$$
\operatorname{dim}_{\mathbb{C}}\left(\operatorname{Hom}\left(\rho, \rho^{\prime}\right)\right)-\operatorname{dim}_{\mathbb{C}}\left(\operatorname{Hom}^{1}\left(\rho, \rho^{\prime}\right)\right)=\left\langle\underline{\operatorname{dim}}(\rho), \underline{\operatorname{dim}}\left(\rho^{\prime}\right)\right\rangle_{Q},
$$

where $\langle,\rangle_{Q}$ is defined in 61 , 63). Let us denote for $\rho \in \operatorname{Rep}_{\mathbb{C}}(Q)$ :

$$
\operatorname{supp}(\rho)=\operatorname{supp}(\underline{\operatorname{dim}}(\rho))=\left\{i \in V(Q) \mid \underline{\operatorname{dim}}_{i}(\rho) \neq 0\right\} .
$$

For $i \in V(Q)$ the simple representation $s_{i}$ is characterized by $\operatorname{supp}\left(s_{i}\right)=\{i\}, \underline{\operatorname{dim}}_{i}\left(s_{i}\right)=1$. Obviously $\left\{s_{i} \mid i \in V(Q)\right\}$ are exceptional objects in $D^{b}\left(\operatorname{Rep}_{\mathbb{C}}(Q)\right)$. One of the representations in the Kronecker pair $\left(\rho, \rho^{\prime}\right)$, which we shall obtain, is among the exceptional objects $\left\{s_{i} \mid i \in V(Q)\right\}$.

It is useful to denote

$$
A, B \subset V(Q) \quad \operatorname{Arr}(A, B)=\{a \in \operatorname{Arr}(Q) \mid s(a) \in A, t(a) \in B\},
$$

$$
\operatorname{Ed}(A, B)=\operatorname{Ed}(B, A)=\operatorname{Arr}(A, B) \cup \operatorname{Arr}(B, A) .
$$

To find Kronecker pairs in $D^{b}\left(\operatorname{Rep}_{\mathbb{C}}(Q)\right)$ we observe first, that for $\rho, \rho^{\prime} \in \operatorname{Rep}_{\mathbb{C}}(Q)$ we have $\operatorname{Hom}^{\leq-1}\left(\rho, \rho^{\prime}\right)=0$ in $D^{b}\left(\operatorname{Rep}_{\mathbb{C}}(Q)\right)$ and

$$
\begin{gathered}
\operatorname{supp}(\rho) \cap \operatorname{supp}\left(\rho^{\prime}\right)=\emptyset \Rightarrow \operatorname{Hom}\left(\rho, \rho^{\prime}\right)=\operatorname{Hom}\left(\rho^{\prime}, \rho\right)=0, \\
\operatorname{dim}_{\mathbb{C}}\left(\operatorname{Hom}^{1}\left(\rho, \rho^{\prime}\right)\right)=\sum_{a \in \operatorname{Arr}\left(\operatorname{supp}(\rho), \operatorname{supp}\left(\rho^{\prime}\right)\right)} \underline{\operatorname{dim}}_{s(a)}(\rho) \underline{\operatorname{dim}}_{t(a)}\left(\rho^{\prime}\right)
\end{gathered}
$$

which follows by (83). Another useful statement is

Lemma 3.35. Let $Q$ be an Euclidean quiver. Then for each $n \in \mathbb{N}$ there exists an exceptional representation $\rho \in \operatorname{Rep}_{\mathbb{C}}(Q)$, s. t. $\underline{\operatorname{dim}}_{v}(\rho) \geq n$ for each $v \in V(Q)$.

Proof. Let $\delta \in \mathbb{N}_{\geq 1}^{V(Q)}$ be the minimal imaginary root of $\Delta_{+}(Q)$, used in the proof of Lemma $3.14{ }^{16}$. One property of $\delta$ is that $\left.{ }_{-}, \delta\right)_{Q}=0$ on $\mathbb{N}^{V(Q)}$, where $(\alpha, \beta)_{Q}=\langle\alpha, \beta\rangle_{Q}+\langle\beta, \alpha\rangle_{Q}$ is the symmetrization of $\langle,\rangle_{Q}$. We find below a vertex $v \in V(Q)$, s. t. $\left\langle 1_{v}, 1_{v}\right\rangle_{Q}=\frac{1}{2}\left(1_{v}, 1_{v}\right)_{Q}=1$, $\left\langle 1_{v}, \delta\right\rangle_{Q} \neq 0$. Then for any $m \in \mathbb{N}$ we have $1=\frac{1}{2}\left(1_{v}+m \delta, 1_{v}+m \delta\right)_{Q}=\left\langle 1_{v}+m \delta, 1_{v}+m \delta\right\rangle_{Q}=1$, $\left\langle 1_{v}+m \delta, \delta\right\rangle_{Q} \neq 0$, hence, for big enough $m, r=1_{v}+m \delta$ is a real positive root $r \in \Delta_{+}(Q)$ with $\langle r, \delta\rangle_{Q} \neq 0,\left\{r_{v} \geq n\right\}_{v \in V(Q)}$. Hence by [18, p. 27] there is an exceptional representation $\rho$ with $\underline{\operatorname{dim}}(\rho)=r$ and the lemma follows.

If $^{17} \Gamma(Q)=\widetilde{A}_{m}, m \geq 1$ : As far as $Q$ is not an oriented cycle then there is a sink $s \in V(Q)$ (i. e. both the arrows touching $s$ end at it). Hence by $(63)\left\langle 1_{s}, 1_{s}\right\rangle_{Q}=-\left\langle 1_{s}, \delta\right\rangle_{Q}=1$.

If $\Gamma(Q)=\widetilde{D}_{m}, m \geq 4, \widetilde{E}_{6}, \widetilde{E}_{7}, \widetilde{E}_{8}$. In [5, fig. (4.13)] are given the coordinates of $\delta$ for all these options for $\Gamma(Q)$. We take $v \in V(Q)$ to be the extending vertex, in [5, fig. (4.13)] this vertex is denoted by $\star$, i.e. $v=\star$. Then by $(63)$ and the given in [5, fig. (4.13)] coordinates of $\delta$ one computes $\left\langle 1_{v}, 1_{v}\right\rangle_{Q}=1,\left\langle 1_{v}, \delta\right\rangle_{Q}= \pm 1$, depending on whether $\star$ is a sink/source in $Q$.

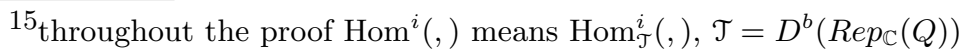

${ }^{16}$ In [5, fig. (4.13)] are given the coordinates of $\delta$ for all euclidean graphs

${ }^{17}$ Recall that by $\Gamma(Q)$ we denote the underlying non-oriented graph and that $\widetilde{A}_{1}$ is graph with two vertices and two parallel edges connecting them, $\widetilde{A}_{m}$ with $m \geq 2$ is a loop with $m+1$ vertices and $m$ edges connecting them forming a simple loop
} 
Definition 3.36. Let $A \subset V(Q), A \neq \emptyset$. By $Q_{A}$ we denote the quiver with $V\left(Q_{A}\right)=A$ and $\operatorname{Arr}\left(Q_{A}\right)=\operatorname{Arr}(A, A)=\{a \in \operatorname{Arr}(Q) \mid s(a) \in A, t(a) \in A\}$. For any $\rho \in \operatorname{Rep}_{\mathbb{C}}\left(Q_{A}\right)$ we denote by the same letter $\rho$ the representation in $\operatorname{Rep}_{\mathbb{C}}(Q)$, which on $A, \operatorname{Arr}(A, A)$ coincides with $\rho$ and is zero elsewhere.

We say that a vertex $v \in V(Q)$ is adjacent to $Q_{A}$ if $v \notin A$ and $\operatorname{Ed}(A,\{v\}) \neq \emptyset$.

Remark 3.37. If $\rho \in \operatorname{Rep}_{\mathbb{C}}\left(Q_{A}\right)$ is an exceptional representation then the corresponding extended representation in $\operatorname{Rep}_{\mathbb{C}}(Q)$ is also exceptional.

Remark 3.38. If $v \in V(Q)$ is adjacent to $Q_{A}$ then $\operatorname{Arr}\left(Q_{A \cup\{v\}}\right)=\operatorname{Arr}\left(Q_{A}\right) \cup \operatorname{Ed}(A,\{v\})$.

In the following two corollaries we consider a configuration of a subset $A \subset V(Q)$ and an adjacent to it vertex $v \in V(Q)$, s. t. the arrows connecting $v$ and $A$ are all directed either from $v$ to $A$ or from $A$ to $v$, which means that $v$ is either a source or a sink in $Q_{A \cup\{v\}}$.

In Corollary 3.39 we show that if $Q_{A}$ is an Euclidean quiver then we get a Kronecker pair $\left(E_{1}, E_{2}\right)$ in $\operatorname{Rep}_{\mathbb{C}}(Q)$ with $\operatorname{dim}_{\mathbb{C}}\left(\operatorname{Hom}^{1}\left(E_{1}, E_{2}\right)\right)$ as big as we want (without additional assumption on the quiver $Q$ ).

In Corollary 3.40 we show that if $\Gamma\left(Q_{A}\right)$ is either $A_{n}(n \geq 1)$ or $D_{n}(n \geq 4)$ then, under the additional assumption that there are at least three edges between $v$ and $A$, we get a Kronecker pair $\left(E_{1}, E_{2}\right)$ in $\operatorname{Rep}_{\mathbb{C}}(Q)$ with $\operatorname{dim}_{\mathbb{C}}\left(\operatorname{Hom}^{1}\left(E_{1}, E_{2}\right)\right)$ equal to this number of edges.

Corollary 3.39. Let $A \subset V(Q)$ be such that $Q_{A}$ is Euclidean. Let $v \in V(Q)$ be a vertex, which is adjacent to $Q_{A}$ and either a sink or a source in $Q_{A \cup\{v\}}$. Then for any $n \geq 3$ there exists a Kronecker pair $\left(E_{1}, E_{2}\right)$ in $\operatorname{Rep}_{\mathbb{C}}(Q)$ with $\operatorname{dim}\left(\operatorname{Hom}^{1}\left(E_{1}, E_{2}\right)\right) \geq n$.

Proof. From Lemma 3.35 and Remark 3.37 we get an exceptional representation $\rho \in \operatorname{Re} p_{\mathbb{C}}(Q)$, s. t. $\operatorname{supp}(\rho)=A$ and $\left\{\underline{\operatorname{dim}}_{i}(\rho) \geq n\right\}_{i \in A}$.

If $v$ is a sink in $Q_{A \cup\{v\}}$ then $\operatorname{Arr}(\{v\}, A)=\emptyset$, and, since $v$ is adjacent to $Q_{A}, \operatorname{Arr}(A,\{v\}) \neq \emptyset$. From $\{v\} \cap A=\emptyset$ and (86) we get $\operatorname{Hom}^{*}\left(s_{v}, \rho\right)=0, \operatorname{Hom}^{\leq 0}\left(\rho, s_{v}\right)=0, \operatorname{dim}_{\mathbb{C}}\left(\operatorname{Hom}^{1}\left(\rho, s_{v}\right)\right) \geq n$. So that $\left(\rho, s_{v}\right)$ is the Kronecker pair we need.

If $v$ is a source in $Q_{A \cup\{v\}}$ then the same arguments show that $\left(s_{v}, \rho\right)$ is such a Kronecker pair.

Corollary 3.40. Let $A \subset V(Q)$ be such that $\Gamma\left(Q_{A}\right)$ is either $A_{n}(n \geq 1)$ or $D_{n}(n \geq 4)$. Let $v \in V(Q)$ be adjacent to $Q_{A}$ and either a sink or a source in $Q_{A \cup\{v\}}$. Let $\#(E d(\{v\}, A))=n \geq 3$. Then there exists a Kronecker pair $\left(E_{1}, E_{2}\right)$ in $\operatorname{Rep}_{\mathbb{C}}(Q)$ with $\operatorname{dim}\left(\operatorname{Hom}^{1}\left(E_{1}, E_{2}\right)\right)=n$.

Proof. Using that $\Gamma\left(Q_{A}\right)$ is either $A_{n}(n \geq 1)$ or $D_{n}(n \geq 4)$ we see that the representation $\rho$, with $A \ni i \mapsto \mathbb{C}, \operatorname{Arr}(A, A) \ni a \mapsto I d_{\mathbb{C}}$ and zero elsewhere is an exceptional representation in $\operatorname{Rep}_{\mathbb{C}}(Q)$ with $\operatorname{supp}(\rho)=A$ and $\left\{\underline{\operatorname{dim}}_{i}(\rho)=1\right\}_{i \in A}$.

If $v$ is a sink in $Q_{A \cup\{v\}}$ then $\operatorname{Arr}(\{v\}, A)=\emptyset$ and $\#(\operatorname{Arr}(A,\{v\}))=\#(E d(\{v\}, A))=n$. From $\{v\} \cap A=\emptyset$ and $(86)$ we get $\operatorname{Hom}^{*}\left(s_{v}, \rho\right)=0, \operatorname{Hom}^{\leq 0}\left(\rho, s_{v}\right)=0, \operatorname{dim}_{\mathbb{C}}\left(\operatorname{Hom}^{1}\left(\rho, s_{v}\right)\right)=n$. So that $\left(\rho, s_{v}\right)$ is the Kronecker pair we need.

If $v$ is a source in $Q_{A \cup\{v\}}$ then the same arguments show that $\left(s_{v}, \rho\right)$ is such a Kronecker pair.

An immediate consequence of this corollary is

Corollary 3.41. If there are $n \geq 3$ parallel arrows in $\operatorname{Arr}(Q)$. Then there exists a Kronecker pair $\left(E_{1}, E_{2}\right)$ in $\operatorname{Rep}_{\mathbb{C}}(Q)$ with $\operatorname{dim}\left(\operatorname{Hom}^{1}\left(E_{1}, E_{2}\right)\right)=n$. 
Proof. Let these arrows start at a vertex $i$ and end at a vertex $j$, then $\# \operatorname{Arr}(\{i\},\{j\})=n \geq 3$, $\operatorname{Arr}(\{j\},\{i\})=\emptyset$ and we apply the previous Corollary to $A=\{j\}, v=\{i\}$.

Whence, we can assume that there are not more than two parallel arrows in $\operatorname{Arr}(Q)$. We consider next the case that two parallel arrows do occur.

Remark 3.42. In the considerations, that follow, we refer most often to Corollary 3.39 (i. $e$. then we get Kronecker pairs with arbitrary big $\operatorname{dim}_{\mathbb{C}}\left(\operatorname{Hom}^{1}\left(E_{1}, E_{2}\right)\right)$ ), but there are three situations, where we need Corollary $3.49 D^{18}$ with the minimal admissible number of edges connecting $v$ and $A$, namely 3 , and then the produced Kronecker pair is with minimal possible $\operatorname{dim}_{\mathbb{C}}\left(\operatorname{Hom}^{1}\left(E_{1}, E_{2}\right)\right)=3$.

The quiver $Q_{A \cup\{v\}}$ observed in these three special situation ${ }^{19}$, in which we use Corollary 3.40 , is as follows (we denote the set $A$ by $A=\left\{a_{1}, a_{2}, \ldots, a_{n}\right\}$ ):
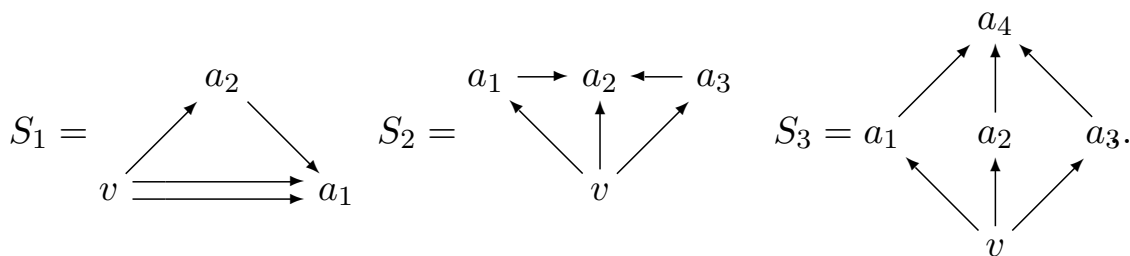

In $S_{1} \Gamma\left(Q_{A}\right)=A_{2}$, in $S_{2} \Gamma\left(Q_{A}\right)=A_{3}$, in $S_{3} \Gamma\left(Q_{A}\right)=D_{4}$.

3.4.1. If there are 2 parallel arrows in $\operatorname{Arr}(Q)$. Let these two arrows start at a vertex $i$ and end at a vertex $j$. So that throughout this subsubsection we have $\#(\operatorname{Arr}(\{i\},\{j\}))=2, \operatorname{Arr}(\{j\},\{i\})=\emptyset$. Then (recall Definition 3.36):

$$
Q_{\{i, j\}}=i \rightrightarrows j, \quad \Gamma\left(Q_{\{i, j\}}\right)=\widetilde{A}_{1} .
$$

By our assumption that $Q$ is connected and not Euclidean there is a vertex $k \in V(Q)$ which is adjacent to $Q_{\{i, j\}}$. If either $\operatorname{Arr}(\{k\},\{i, j\})=\emptyset$ or $\operatorname{Arr}(\{i, j\},\{k\})=\emptyset$ then by Corollary 3.39 we get a Kronecker pair. Hence we can assume $\operatorname{Arr}(\{k\},\{i, j\}) \neq \emptyset$ and $\operatorname{Arr}(\{i, j\},\{k\}) \neq \emptyset$. From the condition that there are not oriented cycles we reduce to

$$
\operatorname{Arr}(\{k\},\{j\}) \neq \emptyset \operatorname{Arr}(\{i\},\{k\}) \neq \emptyset \Rightarrow \operatorname{Arr}(\{j\},\{k\})=\operatorname{Arr}(\{k\},\{i\})=\emptyset .
$$

If $\operatorname{Arr}(\{k\},\{j\})$ has two elements then $Q_{\{k, j\}}=k \rightrightarrows j, \Gamma\left(Q_{\{k, j\}}\right)=\widetilde{A}_{1}, i$ is adjacent to $Q_{\{k, j\}}$ and $\operatorname{Arr}(\{k, j\},\{i\})=\operatorname{Arr}(\{k\},\{i\}) \cup \operatorname{Arr}(\{j\},\{i\})=\emptyset$, hence Corollary 3.39 produces a Kronecker pair. So that we can assume that $\operatorname{Arr}(\{k\},\{j\})$ has only one element.

If $\operatorname{Arr}(\{i\},\{k\})$ has two elements, then $Q_{\{i, k\}}=i \rightrightarrows k, \Gamma\left(Q_{\{i, k\}}\right)=\widetilde{A}_{1}, j$ is adjacent to $Q_{\{i, k\}}$ and $\operatorname{Arr}(\{j\},\{i, k\})=\operatorname{Arr}(\{j\},\{i\}) \cup \operatorname{Arr}(\{j\},\{k\})=\emptyset$, hence Corollary 3.39 produces a Kronecker pair. Hence we can assume that $\operatorname{Arr}(\{i\},\{k\}), \operatorname{Arr}(\{k\},\{j\})$ are single element sets. Using that $Q_{\{i, j\}}=i \rightrightarrows j$ we obtain that $Q_{\{i, j, k\}}$ is the same as the quiver $S_{1}$ in Remark 3.42 with $v=i$, $a_{1}=j, a_{2}=k$ and then by Corollary 3.40 we obtain a Kronecker pair.

We reduce to the case

\footnotetext{
18 we already used it once in Corollary 3.41

19 in Corollary 3.41 the quiver $Q_{A \cup\{v\}}$ is the Kronecker quiver, i. e. $Q_{A \cup\{v\}}=K(n), n \geq 3$
} 
3.4.2. If there are not parallel arrows in $\operatorname{Arr}(Q)$. In this case for any pair $i, j \in V(Q), i \neq j$ we have $\#(\operatorname{Arr}(i, j))=\#(E d(i, j)) \leq 1$. In this subsubsection the term loop with $m$ vertices, $m \geq 1$ in $\Gamma(Q)$ means a sequence $a_{1}, a_{2}, \ldots, a_{m}$ in $V(Q)$, s. t. $\#\left\{a_{1}, a_{2}, \ldots, a_{m}\right\}=m$ and $\left\{E d\left(a_{i}, a_{i+1}\right) \neq \emptyset\right\}_{i=1}^{m-1}$, $E d\left(a_{m}, a_{1}\right) \neq \emptyset$. Since there are not edges-loops and there are not parallel arrows in $Q$, then any loop in $\Gamma(Q)$ (if there is such) must be with $m \geq 3$ vertices.

First we show quickly how to get a Kronecker pair if there are not loops.

If there are not loops in $\Gamma(Q)$ (recall also that by assumption $Q$ is neither Dynkin nor Euclidean) then one can show that for some proper subset $A \subset V(Q) Q_{A}$ is an Euclidean quiver of the type $\widetilde{D}_{m}, m \geq 4, \widetilde{E}_{6}, \widetilde{E}_{7}, \widetilde{E}_{8}$. Take an adjacent to $Q_{A}$ vertex $v \in V(Q)$. The assumption that there are not loops in $\Gamma(Q)$ imply that there is a unique edge between $v$ and $Q_{A}$, i. e. either $\operatorname{Arr}(\{v\}, A)=\emptyset$ or $\operatorname{Arr}(A,\{v\})=\emptyset$, and then we can apply Corollary 3.39 to obtain a Kronecker pair.

Till the end of this subsubsection we assume that there is a loop in $\Gamma(Q)$. Let us fix a loop with minimal number of vertices $a_{1}, a_{2}, \ldots, a_{m}$, i. e. $m$ is the minimal possible number of vertices in a loop. Denote $A=\left\{a_{1}, a_{2}, \ldots, a_{m}\right\}, \#(A)=m$. From the minimality of $m$ it follows that $E d\left(a_{i}, a_{j}\right)=\emptyset$, if $1 \leq i<j \leq m, 2 \leq j-i \leq m-2$, hence $Q_{A}$ (recall Definition 3.36) is a quiver with $\Gamma\left(Q_{A}\right)=\widetilde{A}_{m-1}$. As above, there exists an adjacent to $Q_{A}$ vertex $v \in V(Q)$. From Corollary 3.39 it follows that we can assume $\operatorname{Arr}(\{v\}, A) \neq \emptyset, \operatorname{Arr}(A,\{v\}) \neq \emptyset$. In particular $\#(\operatorname{Ed}(A,\{v\})) \geq 2$. Let us summarize

$$
\begin{gathered}
\Gamma\left(Q_{A}\right)=\widetilde{A}_{m-1}, \quad\{v\} \cap A=\emptyset, \quad \operatorname{Arr}(\{v\}, A) \neq \emptyset, \operatorname{Arr}(A,\{v\}) \neq \emptyset \\
m \geq \#(E d(A,\{v\})) \geq 2, m \geq 3 .
\end{gathered}
$$

We consider several cases depending on the numbers $m$, \#(Ed(A,\{v\})).

The case $\#(E d(A,\{v\}))=m=3$.

We can order $A=\left\{a_{1}, a_{2}, a_{3}\right\}$ so that $Q_{A}=\overbrace{a_{1}}^{a_{2}} a_{3}$. By \# $\# d(A,\{v\}))=3$ it follows that $v \in V(Q)$ must be connected to all the three vertices $\left\{a_{1}, a_{2}, a_{3}\right\}$ and by 89 one of the arrows must start at $v$ and another must end at it. We have either $\operatorname{Arr}\left(\{v\},\left\{a_{2}\right\}\right) \neq \emptyset$ or $\operatorname{Arr}\left(\left\{a_{2}\right\},\{v\}\right) \neq \emptyset$.

If $\operatorname{Arr}\left(\{v\},\left\{a_{2}\right\}\right) \neq \phi$, then by the assumption that there are not oriented cycles we have $\operatorname{Arr}\left(\left\{a_{3}\right\},\{v\}\right)=\phi, \operatorname{Arr}\left(\{v\},\left\{a_{3}\right\}\right) \neq \phi$, so that we can only choose the direction of $\operatorname{Ed}\left(v, a_{1}\right)$, i. e. we have two options for $Q_{\left\{a_{1}, a_{2}, a_{3}, v\right\}}$ :
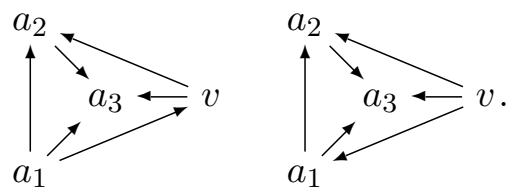

In both the cases $a_{3}$ is a sink in $Q_{\left\{a_{1}, a_{2}, a_{3}, v\right\}}$, hence we can apply Corollary 3.39 to $Q_{\left\{a_{1}, a_{2}, v\right\}}$ and $a_{3} \in V(Q)$ and get Kronecker pairs.

If $\operatorname{Arr}\left(\left\{a_{2}\right\},\{v\}\right) \neq \phi$, now the direction of $E d\left(v, a_{1}\right)$ is fixed and both the options for $Q_{\left\{a_{1}, a_{2}, a_{3}, v\right\}}$ are:
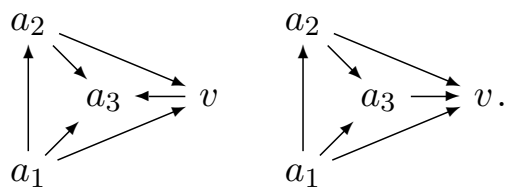
In the first case we apply Corollary 3.39 to $Q_{\left\{a_{1}, a_{2}, v\right\}}$ and $a_{3} \in V(Q)$ and in the second we apply it to $Q_{\left\{a_{1}, a_{2}, a_{3}\right\}}$ and $v \in V(Q)$.

The case $\#(E d(A,\{v\}))=2, m=3$.

Let us consider $\Gamma\left(Q_{A}\right)=\left.\right|_{a_{1}} a_{3}$ without fixing the orientation. Now there are only two edges between $A$ and $v$. Hence by 89 there are exactly two arrows between $A$ and $v$ and one of them must start at $v$ and the other must end at it. As long as we have not fixed the orientations of the arrows in $Q_{A}$, we can assume that $\operatorname{Arr}\left(\left\{a_{1}\right\},\{v\}\right) \neq \emptyset, \operatorname{Arr}\left(\{v\},\left\{a_{2}\right\}\right) \neq \emptyset$. So that $Q_{\left\{a_{1}, a_{2}, a_{3}, v\right\}}$, up to a choice of orientation in $Q_{A}$, is of the arrows in $Q_{A}$. If $a_{3}$ is a source/sink in $Q_{A}$, then it is a source/sink in $Q_{A \cup\{v\}}$ and we can apply Corollary 3.39 to $Q_{\left\{a_{1}, a_{2}, v\right\}}$ and $a_{3} \in V(Q)$. Whence, by the condition that $Q$ is acyclic, we reduce to

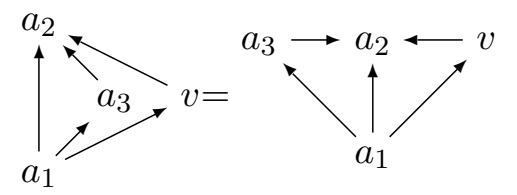

and this is a permutation of the special case $S_{2}$ of Remark 3.42, In this case we obtain a Kronecker pair by Corollary 3.40 applied to $Q_{\left\{v, a_{2}, a_{3}\right\}}$ and $a_{1} \in V(Q)$.

The case $m=4$. $a_{4}-a_{3}$

In this case $\Gamma\left(Q_{A}\right)=\left.\right|_{a_{1} \ldots a_{2}}$ and by the minimality of $m=4$ it follows $\#(\operatorname{Ed}(A,\{v\}))=2($ recall that we have reduced to $(89)$ ). In other words the adjacent vertex $v$ must be connected to two of the vertices of the quadrilateral $\Gamma\left(Q_{A}\right)$. Again by the minimality of $m=4$ these two vertices must be diagonal and, as long as we have not fixed the orientations of the arrows, we can assume that $\operatorname{Arr}\left(\left\{a_{1}\right\},\{v\}\right) \neq \emptyset, \operatorname{Arr}\left(\{v\},\left\{a_{3}\right\}\right) \neq \emptyset$. So that $Q_{\left\{a_{1}, a_{2}, a_{3}, a_{4}, v\right\}}$, up to a choice of orientation in $Q_{A}$, is $a_{4} a_{2} v$. It follows to assign directions of the arrows in $Q_{A}$. If $a_{4}$ or $a_{2}$ is a source/sink in $Q_{A}$ then we can apply Corollary 3.39 to $Q_{\left\{a_{1}, a_{2}, a_{3}, v\right\}}$ and $a_{4} \in V(Q)$ or to $Q_{\left\{a_{1}, a_{4}, a_{3}, v\right\}}$ and $a_{2} \in V(Q)$, respectively. Whence, we reduce to $Q_{\left\{a_{1}, a_{2}, a_{3}, a_{4}, v\right\}}=a_{4} a_{2}$

of the quiver $S_{3}$ in Remark 3.42 .

The case $m \geq 5$. 
If $m=2 k+1$ is odd, $k \geq 2$, then we can depict $\Gamma\left(Q_{A}\right), v \in V(Q)$ and one edge in $E d\left(\{v\}, Q_{A}\right)$ as follows:

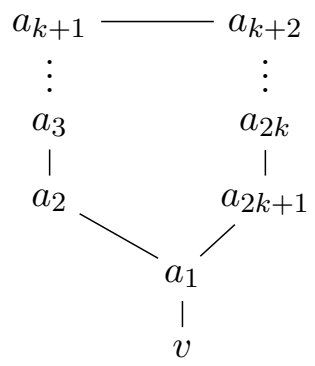

We have reduced to the case $\#\left(E d\left(\{v\}, Q_{A}\right)\right) \geq 2$ (see (89p). If we add another edge between $v$ and $A$ then we obtain another loop with number of vertices less or equal to $k+2$. By $k \geq 2$ we have $k+2<2 k+1$, which contradicts the minimality of $m=2 k+1$.

If $m=2 k$ is even, $k \geq 3$, then we can depict $\Gamma\left(Q_{A}\right), v \in V(Q)$ and one edge in $\operatorname{Ed}\left(\{v\}, Q_{A}\right)$ as follows:

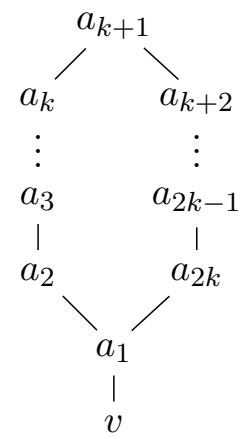

Again, another edge between $v$ and $A$ produces another loop with number of vertices less or equal to $k+2$. By $k \geq 3$ we have $k+2<2 k$, which contradicts the minimality of $m=2 k$.

Proposition 3.34 is completely proved and it implies Proposition 3.32 .

Having Proposition 3.32, Remark 3.33, Corollary 3.15 and Lemma 3.13 we obtain table 1 .

3.5. Further examples of Kronecker pairs. Here we give some more examples of Kronecker pairs. In all the cases Corollary 3.29 can be applied.

3.5.1. Markov triples. It is shown in [11, Example 3.2] that if $X$ is a smooth projective variety (we assume over $\mathbb{C}$ ), such that $D^{b}(\operatorname{Coh}(X))$ is generated by a strong exceptional collection of three elements (for example $X=\mathbb{P}^{2}$ ) then for any such collection $\left(E_{0}, E_{1}, E_{2}\right)$ the dimensions $a=\operatorname{dim}\left(\operatorname{Hom}\left(E_{0}, E_{1}\right)\right), b=\operatorname{dim}\left(\operatorname{Hom}\left(E_{0}, E_{2}\right)\right), c=\operatorname{dim}\left(\operatorname{Hom}\left(E_{1}, E_{2}\right)\right)$ satisfy Markov's equation $a^{2}+b^{2}+c^{2}=a b c$. If $(a, b, c) \neq(0,0,0)$ and $a, b, c \leq 3$ then $(a, b, c)$ satisfy Markov's equation iff $a=b=c=3$, i. e. the "minimal" such triple is $(3,3,3)$. Hence for any strong collection $\left(E_{0}, E_{1}, E_{2}\right)$ on $D^{b}(C o h(X))$ for some $i<j$ the pair $\left(E_{i}, E_{j}[-1]\right)$ is Kronecker. Corollary 3.29 can be applied since $D^{b}(\operatorname{Coh}(X))$ is of finite type.

3.5.2. $\mathbb{P}^{1} \times \mathbb{P}^{1}$. In [42, p. 3] a full exceptional collection consisting of sheaves on $\mathbb{P}^{1} \times \mathbb{P}^{1}$ is described. The matrix given there contains the dimensions of $\operatorname{Hom}\left(E_{i}, E_{j}\right)$, where $E_{i}, E_{j}$ are pairs in the exceptional collection. The number 4 in this matrix corresponds to a Kronecker pair. 
3.5.3. $\mathbb{P}^{n}, n \geq 2$ and their blow ups. Another example, where Corollary 3.29 can be applied, is the standard strong exceptional collection $(\mathcal{O}, \mathcal{O}(1), \ldots, \mathcal{O}(n))$ on $\mathbb{P}^{n}, n \geq 2$. For $n \geq 2\{\operatorname{dim}(\operatorname{Hom}(\mathcal{O}(i-$ $1), \mathcal{O}(i))) \geq 3\}_{i=1}^{n}$ so that $\{(\mathcal{O}(i-1), \mathcal{O}(i)[-1])\}_{i=1}^{n}$ are all Kronecker pairs.

Take now $\mathbb{P}^{n}, n \geq 2$ and blow it up in finite number of points and let the obtained variety be $X$. By [10, Theorem 4.2] we know that $D^{b}(X)$ has a semiorthogonal decomposition $\left\langle E_{1}, E_{2}, \ldots, E_{l}, D^{b}\left(\mathbb{P}^{n}\right)\right\rangle$, where $E_{1}, E_{2}, \ldots, E_{l}$ are exceptional objects. The Kronecker pairs of $D^{b}\left(\mathbb{P}^{n}\right)$ are also Kronecker pairs in $D^{b}(X)$ and Corollary 3.29 can be applied. In particular these arguments hold for all del Pezzo surfaces.

After blowing up in a more general subvarieties $Y \subset \mathbb{P}^{n}$ we still get Kronecker pairs but in this case one must check the extendability condition in Theorem 3.27 (see remark 3.30 ).

\section{Open questions}

In the previous sections we have described the foundations of some new directions in the theory of derived categories and their connection with "classical" geometry and dynamical systems. We believe this is a promising field and conclude by formulating possible future directions of research.

4.1. Algebraicity of entropy. In all examples we have considered so far the graph of the entropy function $t \mapsto h_{t}(F)$ is an algebraic curve defined over $\mathbb{Q}$ in coordinates $\left(\exp (t), \exp \left(h_{t}(F)\right)\right)$. Also, in the $\mathbb{Z} / 2$-graded case $\exp \left(h_{0}(F)\right)$ is an algebraic number. (The stretch factor of a pseudo-Anosov map is an algebraic integer of degree bounded above by a quantity depending only on the genus, see [25].)

Question 4.1. Is algebraicity of entropy a general phenomenon, and if so, what are natural sufficient conditions for it?

4.2. Pseudo-Anosov autoequivalences. Recall that pseudo-Anosov maps are characterized by the fact that they preserve a quadratic differential up to rescaling in the horizontal and vertical direction by factors $1 / \lambda$ and $\lambda$ respectively. Conjecturally, such a quadratic differential defines a stability condition on the (wrapped) Fukaya category of the complement of its zeros. This stability condition would then be preserved by the autoequivalence up to rescaling of the real and imaginary parts of the central charge. In the case of the torus this follows from homological mirror symmetry and the description of the space of stability conditions of the bounded derived category of an elliptic curve. With this in mind, we propose the following.

Definition 4.1. An autoequivalence $\phi$ of a triangulated category $\mathrm{C}$ is pseudo-Anosov if there exists a Bridgeland stability condition $\sigma$ on $\mathcal{C}$ and $\lambda>1$ such that

$$
\phi \sigma=\left(\begin{array}{cc}
\lambda^{-1} & 0 \\
0 & \lambda
\end{array}\right) \sigma
$$

where we use the action of the universal cover of $G L^{+}(2, \mathbb{R})$ on $\operatorname{Stab}(\mathcal{C})$.

An example is given by the shifted Serre functor $S \circ[-1]$ on the bounded derived category of the Kronecker quiver with $m \geq 3$ arrows. A $\sigma$ satisfying 90 lies in the chamber where the set of phases is dense in an arc. The stretch factor is given by

$$
\lambda=\frac{m^{2}+\sqrt{m^{4}-4 m^{2}}}{2}-1
$$

and $\log \lambda$ is the entropy of $S \circ[-1]$. 
Suppose $\mathcal{C}$ is the Fukaya-category of a symplectic manifold $M$ and $\phi$ is a pseudo-Anosov autoequivalence in the above sense.

Question 4.2. Are there, in analogy with the case of surfaces, transverse lagrangian foliations of $M$ which are preserved by some symplectomorphism which induces $\phi$ ?

4.3. Birational maps and dynamical spectrum. Recently a new approach to birational geometry was suggested in the work of Cantat and Lamy [16. In their celebrated work they make a parallel between dynamical theory of Teichmüller space and the Bogomolov-Picard-Manin space associated with an algebraic surface. This connection was taken a step further in the work of Blanc and Cantat [8] where the dynamical spectra of the groups of birational automorphisms of surfaces acting on the Bogomolov-Picard-Manin space were investigated.

In the previous sections we suggested that the space of stablity conditions should play the role of such a categorical Teichmüller space.

Question 4.3. Is there a categorical approach to Bogomolov-Picard-Manin spaces?

First we restrict ourselves to the case $\operatorname{dim}(X)=2$. Let $X$ be a smooth projective surface over $\mathbb{C}$. A birational map $\phi: X \rightarrow X$ does not induce a functor on $D^{b}(X)$ itself, but (assuming it is an isomorphism in codimension one) on the quotient category $D_{(1)}^{b}(X)=D^{b}(X) / S$, where $S$ is the subcategory of complexes with cohomology supported in codimension at least two (see [40]). On the other hand, $\phi$ has a well-defined dynamical degree $\lambda(\phi)$.

Question 4.4. Are these notions of entropy the same, i.e. is it true that $\log \lambda(\phi)=h_{0}\left(\phi^{*}\right)$ ?

In analogy with the dynamical spectrum studied by Blanc and Cantat, one can, quite generally, consider the set entropies of autoequivalences of a triangulated category. This is an invariant which deserves, in our opinion, further study. A first natural question is:

Question 4.5. What is the relation between the dynamical spectrum of $X$ and the entropy spectrum of $D_{(1)}^{b}(X)$ ?

We move now to the case when $\operatorname{dim}(X)>2$. In this case the main question is:

Question 4.6. What is the right analogue of $D_{(1)}^{b}(X)$ and of the categorical Bogomolov-PicardManin space in the case $\operatorname{dim}(X)>2$ ?

Once this question is answered we expect dynamical spectra of these categories to play an important role in the study of birational geometry of $X$. In particular, the rich geometry of Fano manifolds suggest that the dynamical spectrum will contain significant gaps for many non-rational Fano manifolds.

4.4. Complexity and mass. Suppose $\mathcal{T}$ is a triangulated category with stability condition $\sigma$. Let $E \in \mathcal{T}$ be an object with semistable factors $G_{i}$. The mass $m(E)$ of $E$ is by definition $\sum_{i}\left|Z\left(G_{i}\right)\right|$. As with complexity, we can introduce a parameter $t$ and consider

$$
m_{t}(E)=\sum_{i}\left|Z\left(G_{i}\right)\right| \mathrm{e}^{\frac{\phi\left(G_{i}\right)}{\pi} t}
$$

where $\phi\left(G_{i}\right) \in \mathbb{R}$ is the phase of $G_{i}$. Intuitively, $m_{t}(E)$ measures the "size" of $E$, analogous to $\delta_{t}(G, E)$ for a fixed generator $G$. 
Question 4.7. Is $m_{t}$ equivalent to $\delta_{t}\left(G,,_{-}\right)$, in the same sense that the $\delta_{t}\left(G,{ }_{-}\right)$are equivalent for various choices of $G$ ?

We hope to return to this question in future work.

4.5. Questions related to Kronecker pairs and density of phases. The results of Section 3 are a motivation for the following questions.

Recall that in any of the quivers listed in Remark $(3.42)$ we found Kronecker pairs $\left(E_{1}, E_{2}\right)$ with $\operatorname{dim}\left(\operatorname{Hom}^{k}\left(E_{1}, E_{2}\right)\right)=3$ for $k=1$ and 0 for $k \neq 1$. We expect that the first part of following question has a positive answer:

Question 4.8. Do the inequalities $\left\{\operatorname{dim}\left(\operatorname{Hom}^{k}\left(E_{1}, E_{2}\right)\right) \leq 3\right\}_{k \in \mathbb{Z}}$ hold for any exceptional pair $\left(E_{1}, E_{2}\right)$ in any of the quivers listed in Remark (3.42) ?

Determine all the quivers $Q$, s. t. the dimensions $\left\{\operatorname{dim}\left(\operatorname{Hom}^{k}\left(E_{1}, E_{2}\right)\right)\right\}_{k \in \mathbb{Z}}$, where $\left(E_{1}, E_{2}\right)$ vary through all exceptional pairs, are bounded above.

Recall that by Corollary 3.31 these dimensions are strictly smaller than 3 if $Q$ is either Euclidean or a Dynkin quiver, and they are not bounded above if Corollary 3.39 can be applied to $Q$.

We expect that the categories with non-dense behaviour of phases form a "thin" set of categories. Among these, the categories $D^{b}\left(\operatorname{Rep}_{\mathbb{C}}(Q)\right)$ with $Q$ an Euclidean quiver have somehow remarkable behaviour of $P_{\sigma}$ (see the second row of Table 1). So that the next question is:

Question 4.9. Which are the triangulated categories $\mathcal{T}, s$. $t$. for any $\sigma \in \operatorname{Stab}(\mathcal{T})$ the set of phases $P_{\sigma}$ is either finite or has two limit points?

\section{REFERENCES}

[1] M. Abouzaid, On the Fukaya category of higher genus surfaces, Ad. Math. 217 (2008), no. 3, 1192-1235. 14

[2] R. L. Adler, A. G. Konheim, and M. H. McAndrew, Topological Entropy, Trans. AMS 114 (1965), no. 2, $309-319$.

2

[3] M. Auslander, I. Reiten, and S. O. Smalø, Representation theory of Artin algebras, Cambridge University Press, 1997. 12

[4] M. Ballard, D. Favero, and L. Katzarkov, Orlov spectra: bounds and gaps, Invent. Math. 189 (2012), no. 2, 359-430.

[5] M. Barot, Representations of quivers, http://www.math.jussieu.fr/ keller/ictp2006/lecturenotes/barot.pdf 16, 25

[6] M. P. Bellon and C.-M. Viallet, Algebraic Entropy, Commun. Math. Phys. 104 (1999), 425-437.2

[7] A. Bondal and M. Kapranov, Representable functors, Serre functors and mutations, Math. USSR Izvestija, 35 (1990), 519-541 10, 21

[8] J. Blanc and S. Cantat, Dynamical degrees of birational transformations of projective surfaces, arXiv:1307.0361 2. 4.32

[9] A. I. Bondal, Representations of associative algebras and coherent sheaves, Izv. Akad. Nauk SSSR Ser. Mat. 53 (1989), no. 1, 25-44 (Russian); English transl., Math. USSR-Izv. 34 (1990), no. 1, 23-42. 21

[10] A. I. Bondal and D. Orlov, Derived categories of coherent sheaves, Proc. Internat. Congress of Mathematicians vol. II 46-54 (Beijing, 2002) 31

[11] A. I. Bondal and A. E. Polishchuk, Homological properties of associative algebras: the method of helices, Izv. Ross. Akad. Nauk. Ser. Mat. 57 (1993), no. 2, 3-50; English transl. in Russian Acad. Sci. Izv. Math. 42 (1994), no. $2,219-260.30$

[12] A. Bondal and M. Van den Bergh, Generators and representability of functors in commutative and noncommutative geometry, Moscow Mathematical Journal 3 (2003), 1-36.

[13] T. Bridgeland, Stability conditions on triangulated categories, Annals of Math. 166 (2007), 317-345. 2, 14. 15. 17

[14] A. A. Beilinson, J. Bernstein, and P. Deligne, Faisceaux Pervers, Asterisque 100, Soc. Math. de France (1982). 
[15] T. Bridgeland and I. Smith, Quadratic differentials as stability conditions, arXiv:1302.7030 2

[16] S. Cantat and S. Lamy, Normal subgroups of the Cremona group, Acta Mathematica 210 (2013), 31-94. 2, 4, 32

[17] J. Collins and A. Polishchuk, Gluing stability conditions, arXiv:0902.0323 21, 22, 23

[18] W. Crawley-Boevey, Lectures on Representations of Quivers, http://www1.maths.leeds.ac.uk/ pmtwc/quivlecs.pdf 18,25

[19] W. Crawley-Boevey, Exceptional sequences of representations of quivers, in Representations of algebras, Proc. Ottawa 1992, eds V. Dlab and H. Lenzing, Canadian Math. Soc. Conf. Proc. 14 (Amer. Math. Soc., 1993), 117-124. 23

[20] G. Dimitrov and L. Katzarkov, to appear 18, 23

[21] M. Douglas, D-branes, categories and $\mathrm{N}=1$ supersymmetry. Strings, branes, and M-theory, J. Math. Phys. 42 (2001), no. 7, 2818-2843. 2

[22] M Douglas, Dirichlet branes, homological mirror symmetry, and stability, Proceedings of the International Congress of Mathematicians, Vol. III (Beijing, 2002), 395-408, Higher Ed. Press, Beijing, 2002. 2

[23] V. Dlab and Ringel C.M., Eigenvalues of Coxeter transformations and the Gelfand-Kirillov dimension of the preprojective algebras, Proceedings of the AMS 83 (1981), 228-232. 12

[24] A. I. Efimov, Homological mirror symmetry for curves of higher genus, Adv. Math. 230 (2012), no. 2, 493-530. 13

[25] A. Fathi, F. Laudenbach, and V. Poénaru, Travaux de Thurston sur les surfaces, Asterisque 66-67 (1979) 13, 31

[26] S. Friedland, Entropy of algebraic maps, Proceedings of the Conference in Honor of Jean-Pierre Kahane, J. Fourier Anal. Appl. (1995), Special Issue, 215-228. 9

[27] D. Gaiotto, G. Moore, and A. Neitzke, Wall-crossing, Hitchin systems, and the WKB approximation, Adv. Math $234(2013) 2$

[28] D. Galakhov, P. Longhi, T. Mainiero, G. Moore, and A. Neitzke, Wild Wall Crossing and BPS Giants, arxiv: $1305.5454 \mathrm{v} 120$

[29] I. Gelfand and Y. Manin, Methods of Homological algebra, Springer. 21

[30] V. G. Kac, Infinite root systems, representations of graphs and invariant theory, Inventiones mathematicae 56 (1980), 57-92. 16, 20

[31] B. Keller, Calabi-Yau triangulated categories, Trends in representation theory of algebras and related topics, 467-489, 2008. 10, 12

[32] B. Keller and P. Nicolás, Weight structures and simple dg modules for positive dg algebras. Int. Math. Res. Not. IMNR 2013, no. 5, 1028-1078.21

[33] A. King, Moduli of representations of finite dimensional algebras, Quart. J. Math. Oxford Ser. 45 (1994), 515-530. 15

[34] M. Kontsevich and Y. Soibelman, Notes on $A_{\infty}$-algebras, $A_{\infty}$-categories and Non-Commutative Geometry, Homological Mirror Symmetry, Lecture Notes in Physics 757 (2009), 1-67.6

[35] M. Kontsevich and Y. Soibelman, Motivic Donaldson-Thomas invariants: summary of results, Mirror symmetry and tropical geometry, 55-89, Contemp. Math., 527, Amer. Math. Soc., Providence, RI, 2010. 2

[36] R. K. Lazarsfeld, Positivity in algebraic geometry I: Classical setting: line bundles and linear series, Springer, 2004. 11

[37] K. Lefèvre-Hasegawa, Sur les $A_{\infty}$-catégories, Thèse de Doctorat, 2003. 6

[38] V. A. Lunts, Lefschetz fixed point theorems for Fourier-Mukai functors and DG algebras, J. Algebra 256 (2012), 230-256. 8

[39] E. Macri, Some examples of spaces of stability conditions on derived categories, arXiv:math/0411613 21, 22, 23

[40] S. Meinhardt and H. Partsch, Quotient categories, stability conditions, and birational geometry, arXiv:0805.0492 32

[41] D. Orlov, Remarks on generators and dimensions of triangulated categories, Moscow Math. J., vol. 9, (2009), no. 1, 153-159. 10

[42] M. Perling, Some quivers describing the derived categories of the toric del Pezzos, http://www.mathematik.unikl.de/ perling/quivers.pdf 30

[43] M. Reineke, Moduli of Representations of Quivers, Trends in representation theory of algebras and related topics, 589-637, EMS Ser. Congr. Rep., Eur. Math. Soc., Zürich, 2008. 20

[44] J. Rickard and R. Rouqier, Stable categories and reconstruction, arXiv:1008.1976 21 
[45] C. M. Ringel, Indecomposable representations of the Kronecker quivers, Proc. Amer. Math. Soc. 141 (2013), no. $1,115-121.20$

[46] Seidel P., Vanishing cycles and mutation, European Congress of Mathematics Vol II (Barcelona 2000), Progr. Math. 202, Birkhäuser, 2001, 65-85.

[47] P. Seidel, Homological mirror symmetry for the genus two curve, J. Algebraic Geom. 20 (2011), no. 4, $727-769$. 13

[48] Ya. G. Sinai, On the Notion of Entropy of a Dynamical System, Doklady RAS 124 (1959), 768-771. 2

[49] W. P. Thurston, On the geometry and dynamics of diffeomorphisms of surfaces, AMS Bulletin, New Series 19 (1988), no. 2, 417-413. 13

[50] T. Weist, On the Euler characteristic of Kronecker moduli spaces, arXiv:1203.2740 20

[51] T. Weist, Localization in quiver moduli spaces, Represent. Theory 17 (2013), 382-425. 20

(George Dimitrov) Universität Wien, Garnisongasse 3, 1090, Wien, Österreich

E-mail address: gkid@abv.bg

(Fabian Haiden) Universität Wien, Garnisongasse 3, 1090, Wien, Österreich

E-mail address: fabian.haiden@univie.ac.at

(Ludmil Katzarkov) Universität Wien, Garnisongasse 3, 1090, Wien, Österreich

E-mail address: lkatzark@math.uci.edu

(Maxim Kontsevich) IHES, G35 route De Chartres, F-91440, France

E-mail address: maxim@ihes.fr 